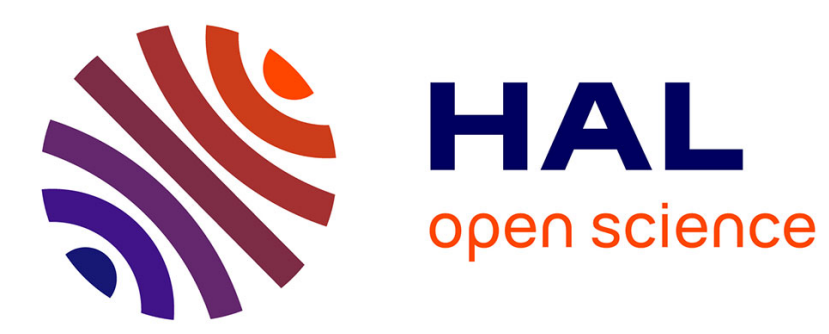

\title{
An extended finite element method approach for structural-acoustic problems involving immersed structures at arbitrary positions
}

Antoine Legay

\section{- To cite this version:}

Antoine Legay. An extended finite element method approach for structural-acoustic problems involving immersed structures at arbitrary positions. International Journal for Numerical Methods in Engineering, 2012, 93, pp.376 - 399. 10.1002/nme.4388 . hal-01422330

\section{HAL Id: hal-01422330 \\ https://hal.science/hal-01422330}

Submitted on 25 Dec 2016

HAL is a multi-disciplinary open access archive for the deposit and dissemination of scientific research documents, whether they are published or not. The documents may come from teaching and research institutions in France or abroad, or from public or private research centers.
L'archive ouverte pluridisciplinaire HAL, est destinée au dépôt et à la diffusion de documents scientifiques de niveau recherche, publiés ou non, émanant des établissements d'enseignement et de recherche français ou étrangers, des laboratoires publics ou privés. 


\title{
An extended finite element method approach for structural-acoustic problems involving immersed structures at arbitrary positions
}

\author{
Antoine Legay \\ Structural Mechanics and Coupled Systems Laboratory, Conservatoire National des Arts et Métiers, \\ 292 rue Saint-Martin, 75141 Paris Cedex 03, France
}

\begin{abstract}
Noise reduction for passengers' comfort in transport industry is now an important constraint to be taken into account during the design process. This process involves to study several configurations of the structures immersed in a given acoustic cavity in the context of an optimization, uncertainty, or reliability study for instance. The finite element method may be used to model this coupled fluid-structure problem but needs an interface conforming mesh for each studied configuration that may become time consuming. This work aims at avoiding this remeshing step by using noncompatible meshes between the fluid and the structures. The immersed structures are supposed to be thin shells and are localized in the fluid domain by a signed distance level-set. To take into account the pressure discontinuity from one side of the structures to the other one, the fluid pressure approximation is enriched according to the structures positions by a Heaviside function using a partition of unity strategy (extended finite element method). The same fluid mesh of the empty cavity is then used during the whole parametric study. The method is implemented for a three-dimensional fluid and tested on academic examples before being applied to an industrial-like case.
\end{abstract}

KEY WORDS: fluid-structure interaction; structural-acoustic; XFEM; parametric study

\section{INTRODUCTION}

Noise reduction of passenger acoustic compartment in transport industry is taking more and more importance in the final characteristics of a vehicle. Undeniably, passengers expect to travel with an increasing comfort whether it is by car, train, or plane. Therefore, this constraint has to be taken into account by the engineers in the design process to reach the set noise level in the passenger compartment.

It is shown in [1] that the seats in an aircraft cabin have significant influences on both the amplitude of the sound pressure level and the resonant frequency shifts. To predict a realistic noise mapping and level in the cavity, the seats and all the significant flexible and absorbing structures have to be taken into account.

This elasto-acoustic problem can be modeled and numerically computed. The finite element method can be used, and a mesh has then to be produced. Classically, this mesh is compatible at the interfaces between the fluid and the structures. During the design step, several configurations are tested to optimize the design, to perform an uncertainty study, or to compute a reliability level. Moreover, a same passenger compartment could be developed into several uses: the same 
car can have back seats or not, and an aircraft can have small seats or large seats depending on the class. For each configuration, a compatible mesh at the fluid-structure interfaces is needed. This remeshing step may become a waste of time for the engineers if a parametric meshing software is not available and if a large number of configurations have to be tested. Moreover, the engineer in charge of the study may possess only the fluid mesh of the empty cavity and so may not be able to remesh it according to the structures. A possible alternative is the use of the boundary element method; however, it suffers the same drawback. Indeed, a new surface mesh would have to be built for each configuration leading to an update of the corresponding full matrices.

The aim of this work is to be able to efficiently analyze different configurations of immersed structures in the acoustic domain and their influences on the noise level in the cavity. The basic idea of the proposed method is to always use the same fluid mesh of the empty cavity for all the possible configurations, assuming that the cavity's geometry does not change. The structures are then arbitrarily immersed within the acoustic fluid mesh. The embedded structures in the fluid are considered to be thin shells. Therefore, the pressure is discontinuous from one side of the structure to the other one. Although this discontinuity is naturally described in the case of a compatible mesh, it is introduced in this work for noncompatible meshes by using a partition of unity based approach [2] where the enrichment is a Heaviside function (XFEM, extended finite element method).

The XFEM was first developed to model cracks [3] and inclusions $[4,5]$ in solid mechanics. It has been applied to several kind of problems where an evolving discontinuity has to be taken into account as in [6] for biofilm growth for instance. The XFEM has already been used in the context of nonlinear fluid-structure interaction for structures embedded in a fluid flow [7-9].

Other techniques such as immersed element method-like are developed to deal with noncompatible meshes for a fluid-structure interaction problem [10-13]. However, these approaches need either to have a thick solid structure or to refine the mesh around the interface to have good results.

The structure is considered to have small displacements, so the discontinuity surface does not evolve with time in the fluid domain. However, for a parametric study of the structure positions in the cavity, and eventually their geometries, the discontinuities move according to the chosen parameters, whereas the acoustic cavity keeps the same geometry. It is assumed that there is fluid on both sides of the structure. The case where the fluid is only on one side of the structure can be performed using a compatible mesh with no change of the fluid mesh. If such a structure is supposed to change its configuration, the fluid mesh has to be build a priori such that all configurations are compatible to avoid remeshing during the parametric study.

No damping is considered in this work for simplicity reasons of implementation. Nevertheless, a structural-acoustic mechanical problem is closely linked to it if the final aim is to reduce noise level in the passenger cavity. This noise level reduction can be performed by several different techniques:

- A porous material can be added on the walls [14], and the resulting numerical problem may be reduced by subdomain decomposition $[15,16]$.

- The absorbing material can be modeled as rheological devices [17-19] to simplify its numerical treatment.

- A viscoelastic material may be put on (or inside) the structure [20], and special numerical strategies can be used to efficiently compute the complex coupled problem [21,22].

These different techniques can be used in addition to the approach developed in this work.

The second section of the paper states the equations of the coupled fluid-structure problem. The third section focuses on the discretization, especially the XFEM approximation. The fourth section gives details of the algorithm as well as the numerical implementation of the method. The last section shows the accuracy of the method by comparing the results to reference solutions and demonstrates the efficiency of the approach to compute different configurations of an industriallike problem. 


\section{STRONG AND WEAK FORMS OF AN ELASTO-ACOUSTIC PROBLEM}

\subsection{Description of the problem}

A fluid-structure interaction problem is considered (Figure 1). The acoustic fluid domain and the thin flexible structures are respectively denoted by $\Omega_{\mathrm{F}}$ and $\Omega_{\mathrm{S}}$. The problem is studied in the frequency domain for a permanent harmonic response at angular frequency $\omega$. The compressible fluid domain is described using pressure $(p)$ as primary variable, whereas displacement $\left(\mathbf{u}_{\mathrm{S}}\right)$ is used for the structural part. Their boundaries are separated into contours of

- imposed Dirichlet boundary conditions denoted $\partial_{1} \Omega_{\mathrm{F}}$ and $\partial_{1} \Omega_{\mathrm{S}}$,

- prescribed Neumann boundary conditions denoted $\partial_{2} \Omega_{\mathrm{F}}$ and $\partial_{2} \Omega_{\mathrm{S}}$, and

- coupling interface between acoustic fluid and structures denoted $\Gamma$.

The thin structures are considered with no thickness in the fluid domain meaning that the structures are seen by the fluid as surfaces. These surfaces are described in the fluid domain by the zero contour of a level-set $\phi(M)$ that is the signed distance to the closest structure (Figure 2). By denoting $d$ the distance of a point $M$ to the structure, the level-set can be defined as

$$
\phi(M)= \pm d,
$$

where $d$ is the minimal distance to the structure; the sign is determined using a conventional unit normal vector $\vec{n}$ pointing arbitrarily outward of one side of the structure. For simplicity reasons, only one structure is considered in the following of this section.

\subsection{Strong form of the equations}

2.2.1. Structure $\left(\boldsymbol{u}_{\mathrm{S}}\right)$. The structural domain is assumed satisfying the elastodynamic linearized equation at angular frequency $\omega$ :

$$
\operatorname{div} \boldsymbol{\sigma}_{\mathrm{S}}+\omega^{2} \rho_{\mathrm{S}} \mathbf{u}_{\mathrm{S}}=\mathbf{0} \text { in } \Omega_{\mathrm{S}},
$$

where $\rho_{\mathrm{S}}$ is the constant density per unit volume and $\sigma_{\mathrm{S}}$ is the Cauchy stress tensor, given by

$$
\boldsymbol{\sigma}_{\mathrm{S}}=\mathbf{D}_{\mathrm{S}} \boldsymbol{\varepsilon}\left(\mathbf{u}_{\mathrm{S}}\right) \text { in } \Omega_{\mathrm{S}},
$$

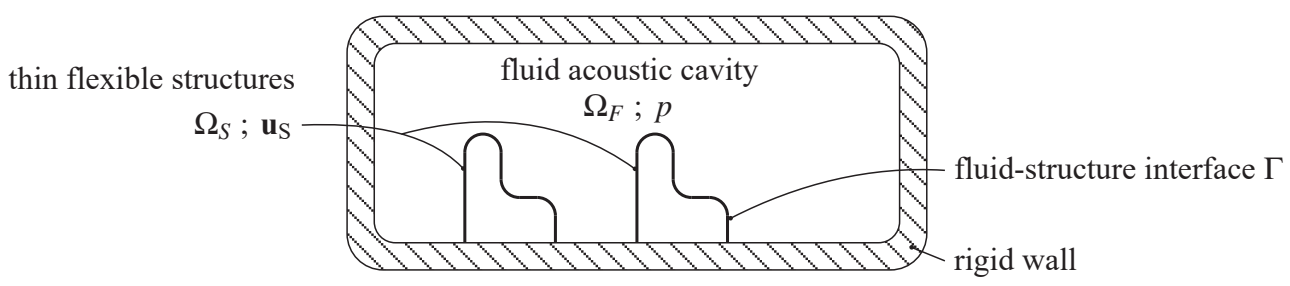

Figure 1. Description and notations of the elasto-acoustic interaction problem.

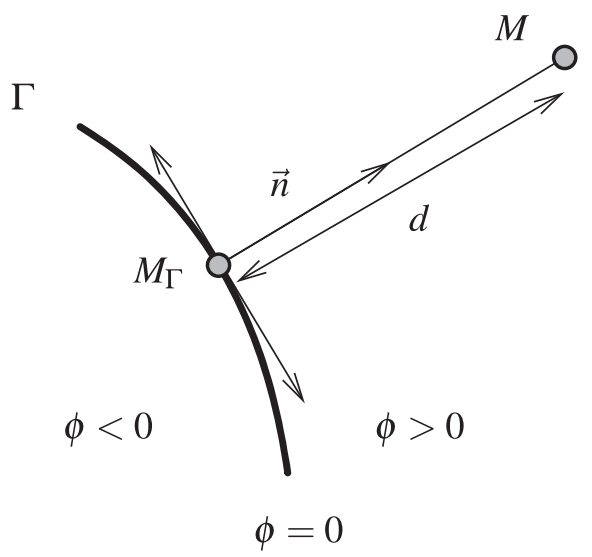

Figure 2. Signed distance to the thin structure. 
where $\mathbf{D}_{\mathrm{S}}$ is the classical Hooke's linear elasticity tensor and $\boldsymbol{\varepsilon}\left(\mathbf{u}_{\mathrm{S}}\right)$ is the strain tensor associated to the displacement vector field $\mathbf{u}_{\mathrm{S}}$, defined by

$$
\boldsymbol{\varepsilon}\left(\mathbf{u}_{\mathrm{S}}\right)=\frac{1}{2}\left(\operatorname{grad} \mathbf{u}_{\mathrm{S}}+\operatorname{grad}^{T} \mathbf{u}_{\mathrm{S}}\right) .
$$

Prescribed displacements and forces are imposed on the external structural boundaries $\partial_{1} \Omega_{\mathrm{S}}$ and $\partial_{2} \Omega_{\mathrm{S}}$, respectively:

$$
\begin{gathered}
\mathbf{u}_{\mathrm{S}}=\mathbf{0} \quad \text { on } \partial_{1} \Omega_{\mathrm{S}} \\
\boldsymbol{\sigma}_{\mathrm{S}} \mathbf{n}_{\mathrm{S}}=\mathbf{f}_{\mathrm{Sb}} \quad \text { on } \partial_{2} \Omega_{\mathrm{S}},
\end{gathered}
$$

where $\mathbf{n}_{\mathrm{S}}$ is the unit normal vector pointing outward $\Omega_{\mathrm{S}}$.

2.2.2. Compressible fluid ( $p$ ). The fluid is assumed compressible and inviscid, and satisfies the classical Helmholtz equation derived from the motion, continuity, and constitutive equations:

$$
\Delta p+\frac{\omega^{2}}{c_{0}^{2}} p=0 \quad \text { in } \Omega_{\mathrm{F}},
$$

where $c_{0}$ is the constant speed of sound in the fluid and $p$ the pressure fluctuation scalar field. The pressure fluctuation is named pressure in the following for simplicity reasons. The limit case where $\omega=0$ (static solution) is not given by Equation (7); this case is not considered in this work; more details are given in [23].

The fluid displacement vector, denoted by $\mathbf{u}_{\mathrm{F}}$, can be related to the pressure field using the linearized Euler equation expressed for a harmonic solution:

$$
\operatorname{grad} p=\omega^{2} \rho_{\mathrm{F}} \mathbf{u}_{\mathrm{F}} \quad \text { in } \Omega_{\mathrm{F}},
$$

where $\rho_{\mathrm{F}}$ is the fluid density.

At external boundary of the acoustic fluid domain, rigid cavity conditions are imposed by setting a free pressure field. An harmonic excitation is prescribed via an acoustic source:

$$
\mathbf{u}_{\mathrm{F}} \cdot \mathbf{n}_{F}=u_{\mathrm{Fb}} \quad \text { on } \partial_{2} \Omega_{\mathrm{F}},
$$

where $\mathbf{n}_{F}$ is the unit normal vector pointing outward the fluid domain and $u_{\mathrm{Fb}}$ is set to zero out of the acoustic source included in $\partial_{2} \Omega_{\mathrm{F}}$.

2.2.3. Fluid-structure coupling. At interface $\Gamma$ between the structural domain and the acoustic fluid, normal stress and normal displacement continuity conditions are given by

$$
\begin{gathered}
\sigma_{\mathrm{S}} \mathbf{n}_{S}-p \mathbf{n}_{F}=\mathbf{0} \text { on } \Gamma, \\
\left(\mathbf{u}_{\mathrm{F}}-\mathbf{u}_{\mathrm{S}}\right) \cdot \mathbf{n}_{F}=0 \text { on } \Gamma .
\end{gathered}
$$

The fluid displacement $\mathbf{u}_{\mathrm{F}}$ is related to the pressure field using Equation (8).

\subsection{Weak form}

The test-function method is used to derive the variational formulation of the coupled problem. For this purpose, the spaces of sufficiently smooth functions $C_{\mathbf{u}_{\mathrm{S}}}$ and $C_{p}$ are introduced, associated to the field variables $\mathbf{u}_{\mathrm{S}}$ and $p$, respectively.

- Let $\delta \mathbf{u}_{\mathrm{S}}$ be the frequency-independent test function, associated to $\mathbf{u}_{\mathrm{S}}$, belonging to the admissible space $C_{\mathbf{u}_{\mathrm{S}}}^{*}=\left\{\delta \mathbf{u}_{\mathrm{S}} \in C_{\mathbf{u}_{\mathrm{S}}} \mid \delta \mathbf{u}_{\mathrm{S}}=\mathbf{0}\right.$ on $\left.\partial_{1} \Omega_{\mathrm{S}}\right\}$. Multiplying Equation (2) combined with 
Equation (3) by $\delta \mathbf{u}_{\mathrm{S}} \in C_{\mathbf{u}_{\mathrm{S}}}^{*}$, applying a Green's formula, and taking Equations (6) and (10) into account lead to

$$
\int_{\Omega_{\mathrm{S}}} \mathbf{D}_{\mathrm{S}} \boldsymbol{\varepsilon}\left(\delta \mathbf{u}_{\mathrm{S}}\right): \boldsymbol{\varepsilon}\left(\mathbf{u}_{\mathrm{S}}\right) \mathrm{d} V-\omega^{2} \int_{\Omega_{\mathrm{S}}} \rho_{\mathrm{S}} \delta \mathbf{u}_{\mathrm{S}} \cdot \mathbf{u}_{\mathrm{S}} \mathrm{d} V-\int_{\Gamma} \delta \mathbf{u}_{\mathrm{S}} \cdot \mathbf{n}_{F} p \mathrm{~d} \Sigma=\int_{\partial_{2} \Omega_{\mathrm{S}}} \delta \mathbf{u}_{\mathrm{S}} \cdot \mathbf{f}_{\mathrm{Sb}} \mathrm{d} \Sigma .
$$

- Let $\delta p$ be the frequency-independent test function, associated to $p$, belonging to the admissible space $C_{p}$. Multiplying Equation (7) by $\delta p \in C_{p}$, applying a Green's formula, and taking Equations (11) and (9) into account lead to

$$
\frac{1}{\rho_{\mathrm{F}}} \int_{\Omega_{\mathrm{F}}} \operatorname{grad} \delta p \cdot \operatorname{grad} p \mathrm{~d} V-\frac{\omega^{2}}{c_{0}^{2} \rho_{\mathrm{F}}} \int_{\Omega_{\mathrm{F}}} \delta p p \mathrm{~d} V-\omega^{2} \int_{\Gamma} \delta p \mathbf{u}_{\mathrm{S}} \cdot \mathbf{n}_{F} \mathrm{~d} \Sigma=\omega^{2} \int_{\partial_{2} \Omega_{\mathrm{F}}} \delta p u_{\mathrm{Fb}} \mathrm{d} \Sigma .
$$

\section{DISCRETIZATION OF THE COUPLED PROBLEM}

\subsection{Structure}

The discrete Kirchhoff triangle element [24-27] is used to model and discretize the thin shell structure (Figure 3(a)). This element is based on the Kirchhoff-Love theory. There are six DOFs, three translations, and three rotations in the global coordinate system. The structure DOFs are collected in a vector $\mathbf{U}_{\mathrm{S}}$ that is linked to the structural displacement $\mathbf{u}_{\mathrm{S}}$ by

$$
\mathbf{u}_{\mathrm{S}}=N_{S} \mathbf{U}_{\mathrm{S}},
$$

where $N_{S}$ is the shape function matrix at the global level including a transformation matrix from local to global coordinates. The structure stiffness matrix and mass matrix are respectively denoted by $\mathbf{K}_{\mathrm{SS}}$ and $\mathbf{M}_{\mathrm{SS}}$.

\subsection{Fluid}

3.2.1. Standard part of the approximation. The tridimensional fluid domain is discretized by fournode tetrahedral elements (Figure 3(b)). The pressure fluctuation $p$ is linked to the node pressure vector $\mathbf{P}$ by

$$
p=N_{F} \mathbf{P},
$$

where $N_{F}$ is the shape function matrix whereas the pressure gradient is given by

$$
\operatorname{grad} p=\boldsymbol{B}_{F} \mathbf{P}
$$

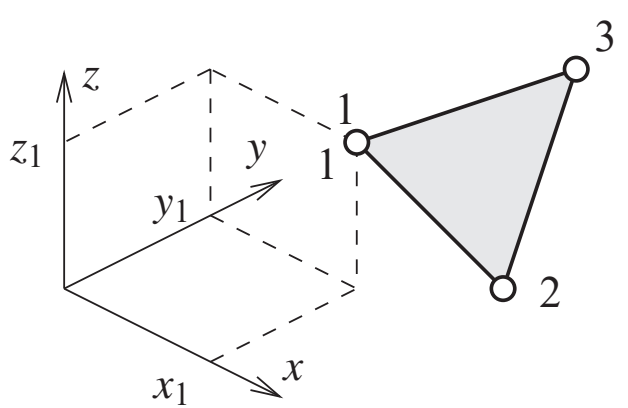

(a) 3-node DKT triangle shell element

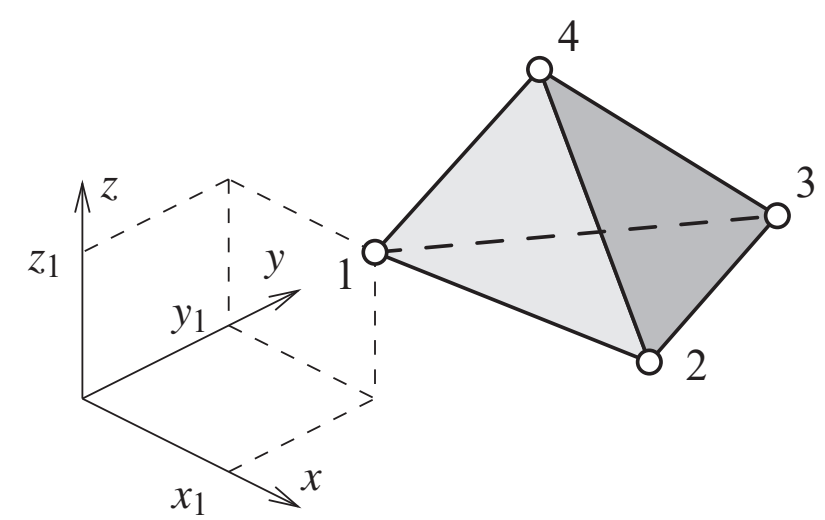

(b) 4-node tetrahedral fluid element

Figure 3. Fluid and structure finite elements. (a) Three-node discrete Kirchhoff triangle shell element; (b) four-node tetrahedral fluid element. 
where $\boldsymbol{B}_{F}$ is the discretized gradient operator. For this element, the constant discretized gradient operator makes easier the integration through the element.

3.2.2. Enrichment of the pressure field. A thin structure placed in a fluid separates locally the fluid into two parts, meaning that the pressure is discontinuous from one side of the structure to the other one (Figure 4(a)). The classical discretization strategy is to make the different meshes compatible at the interface (Figure 4(b)) where three nodes are placed at the same geometrical point: two nodes for each fluid domain and one node for the structure. The discontinuous pressure can be represented naturally by the fluid discretization but needs a remeshing or a mesh-moving technique for each configuration of the structures in the fluid domain. This method is used in this work to compute reference solutions in the application section. In the context of a parametric study of the positions of the structures, this remeshing step can become heavy and difficult to realize.

The alternative strategy used in this work is to have the structure arbitrarily placed in the fluid mesh of the empty cavity (Figure 4(c)): there is no need to remesh the fluid for each structure configuration. To correctly catch the pressure jump from one side of the structure to the other one, the pressure field approximation is enriched by a Heaviside function using a partition of unity strategy [2], namely XFEM [3]. The pressure approximation becomes

$$
p(M)=\sum_{i \in \mathscr{F}} N_{F}^{i}(M) P_{i}+\sum_{i \in \mathscr{A}} N_{F}^{i}(M) \psi_{i}(M) A_{i},
$$

where $\mathscr{F}$ is the set of nodes of the whole mesh, $N_{F}^{i}(M)$ is the shape function associated to the fluid node $i, P_{i}$ is the standard nodal pressure value, $\mathscr{A}$ is the set of enriched nodes, $\psi_{i}(M)$ is the enrichment function, and $A_{i}$ is the additional nodal unknown coming from the enrichment. The set of enriched nodes are those that are connected to the elements cut by the structures (Figure 5) or, in other words, cut by the zero contour of the level-set. The enrichment function is chosen such as

$$
{ }_{i}(M)=H(\phi(M))
$$

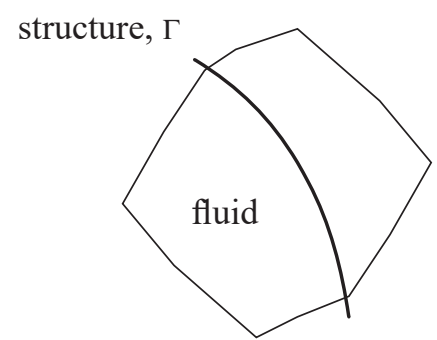

(a) Fluid divided into 2 parts by the structure

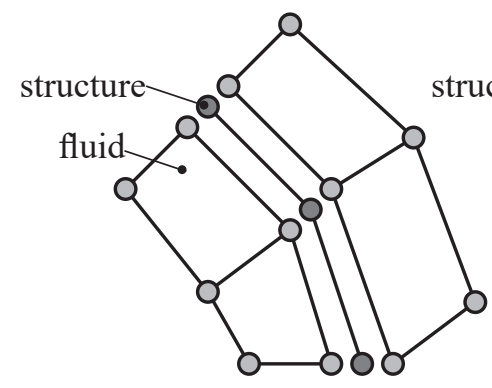

(b) Compatible mesh

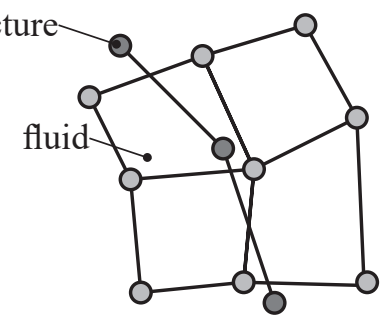

(c) Incompatible mesh

Figure 4. Different ways of meshing the interface. (a) Fluid divided into two parts by the structure; (b) compatible mesh; (c) incompatible mesh.

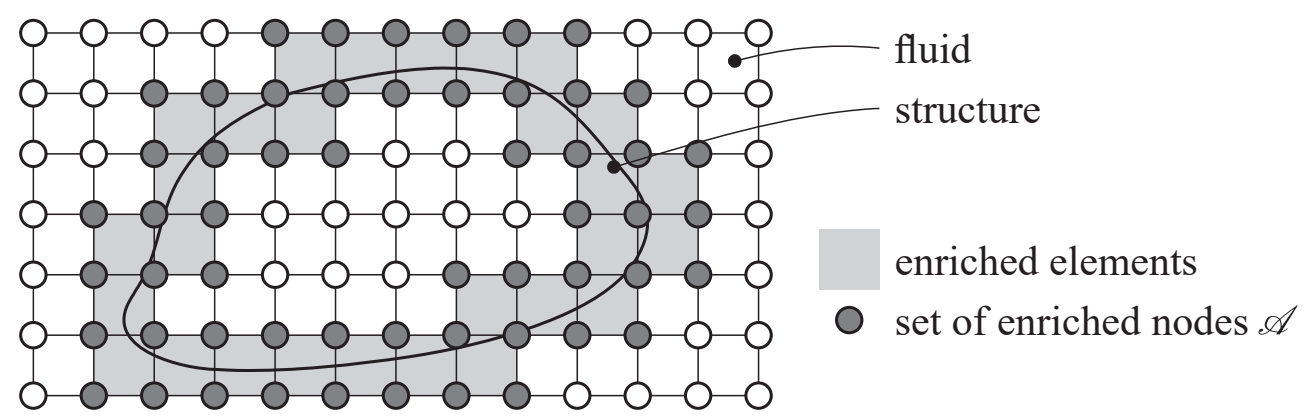

Figure 5. Enriched elements, partition of unity support. 
where $H$ is the Heaviside function and $\phi(M)$ is the signed distance function. The Heaviside function is modified to be the sign of the level-set. The pressure approximation can be written:

$$
p(M)=\sum_{i \in \mathscr{F}} N_{F}^{i}(M) P_{i}+H(\phi(M)) \sum_{i \in \mathscr{A}} N_{F}^{i}(M) A_{i}
$$

because the enrichment function is not node dependant. The full approximation containing both the standard part and the enrichment can be written from Equation (19) as

$$
p=N_{F} \mathbf{P}+N_{A} \mathbf{A}
$$

where

$$
N_{A}(M)=H(\phi(M)) N_{F}(M) \boldsymbol{\beta}_{F A} .
$$

The Boolean localization matrix $\boldsymbol{\beta}_{F A}$ is used to extract enriched set of nodes $\mathscr{A}$ from the set of nodes $\mathscr{F}$. The pressure value at an enriched node $i \in \mathscr{A}$ is then

$$
p\left(M_{i}\right)=P_{i}+H\left(\phi\left(M_{i}\right)\right) A_{i} .
$$

To keep the property of having $p\left(M_{i}\right)=P_{i}$, the enrichment may be chosen as $\psi_{i}(M)=$ $H(\phi(M))-H\left(\phi\left(M_{i}\right)\right)$. However, this shift is not used in this work to make the integration of an enriched element easier.

The level-set function is discretized using the fluid mesh, so $\phi(M)$ is defined as

$$
\phi(M)=\sum_{i \in \mathscr{F}} N_{F}^{i}(M) \phi_{i}
$$

where $\phi_{i}$ is the nodal value of $\phi(M)$.

3.2.3. Fluid-structure coupling. The coupling term in the structural weak form (12) is given by

$$
\int_{\Gamma} \delta \mathbf{u}_{\mathrm{S}} \cdot \mathbf{n}_{F} p \mathrm{~d} \Sigma
$$

It is recalled that in this work, it is assumed that there is fluid on both sides of the structure. Therefore, the interface $\Gamma$ is decomposed into $\Gamma^{+}$and $\Gamma^{-}$, and a point $M_{\Gamma}$ belonging to the interface is split into $M_{\Gamma^{+}}$and $M_{\Gamma^{-}}$(Figure 6):

- $\Gamma^{+}$is the structure surface in contact with the fluid domain where $\phi\left(M_{\Gamma^{+}}\right)>0$ :

。 the unit normal vector is denoted by $\mathbf{n}_{F}^{+}$(with $\mathbf{n}_{S}^{+}=-\mathbf{n}_{F}^{+}$)

○ the Heaviside function $H\left(\phi\left(M_{\Gamma^{+}}\right)\right)$is +1

- $\Gamma^{-}$is the structure surface in contact with the fluid domain where $\phi\left(M_{\Gamma^{-}}\right)<0$ :

。 the unit normal vector is denoted by $\mathbf{n}_{F}^{-}$with $\mathbf{n}_{F}^{-}=-\mathbf{n}_{F}^{+}$

$\circ$ the Heaviside function $H\left(\phi\left(M_{\Gamma^{-}}\right)\right)$is -1

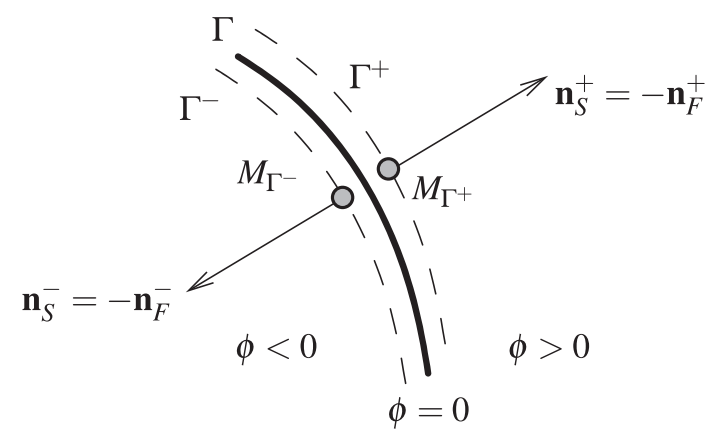

Figure 6. Interface coupling details. 
With the use of these notations, the coupling term is developed as

$$
\begin{aligned}
\int_{\Gamma} \delta \mathbf{u}_{\mathrm{S}} \cdot \mathbf{n}_{F} p \mathrm{~d} \Sigma= & \delta \mathbf{U}_{\mathrm{S}}^{T} \int_{\Gamma} \boldsymbol{N}_{S}^{T} \mathbf{n}_{F} \boldsymbol{N}_{F} \mathrm{~d} \Sigma \mathbf{P}+\delta \mathbf{U}_{\mathrm{S}}^{T} \int_{\Gamma} H(M) N_{S}^{T} \mathbf{n}_{F} \boldsymbol{N}_{F} \mathrm{~d} \Sigma \boldsymbol{\beta}_{F A} \mathbf{A} \\
= & \delta \mathbf{U}_{\mathrm{S}}^{T} \int_{\Gamma^{+}} \boldsymbol{N}_{S}^{T} \mathbf{n}_{F}^{+} \boldsymbol{N}_{F} \mathrm{~d} \Sigma \mathbf{P}+\delta \mathbf{U}_{\mathrm{S}}^{T} \int_{\Gamma^{+}} \boldsymbol{N}_{S}^{T} \mathbf{n}_{F}^{+} \boldsymbol{N}_{F} \mathrm{~d} \Sigma \boldsymbol{\beta}_{F A} \mathbf{A} \\
& +\delta \mathbf{U}_{\mathrm{S}}^{T} \int_{\Gamma^{-}} \boldsymbol{N}_{S}^{T} \mathbf{n}_{F}^{-} \boldsymbol{N}_{F} \mathrm{~d} \Sigma \mathbf{P}-\delta \mathbf{U}_{\mathrm{S}}^{T} \int_{\Gamma^{-}} \boldsymbol{N}_{S}^{T} \mathbf{n}_{F}^{-} \boldsymbol{N}_{F} \mathrm{~d} \Sigma \boldsymbol{\beta}_{F A} \mathbf{A} .
\end{aligned}
$$

Because $\mathbf{n}_{F}^{-}=-\mathbf{n}_{F}^{+}$, the coupling term becomes

$$
\int_{\Gamma} \delta \mathbf{u}_{\mathrm{S}} \cdot \mathbf{n}_{F} p \mathrm{~d} \Sigma=\delta \mathbf{U}_{\mathrm{S}}^{T} 2 \int_{\Gamma} \boldsymbol{N}_{S}^{T} \mathbf{n}_{F}^{+} \boldsymbol{N}_{F} \mathrm{~d} \Sigma \boldsymbol{\beta}_{F A} \mathbf{A} .
$$

The coupling between fluid and structure is only carried out by the fluid pressure enrichment; the coupling matrix $\mathbf{C}_{\mathrm{SA}}$ is defined by

$$
\mathbf{C}_{\mathrm{SA}}=2 \int_{\Gamma} \boldsymbol{N}_{S}^{T} \mathbf{n}_{F}^{+} \boldsymbol{N}_{F} \mathrm{~d} \Sigma \boldsymbol{\beta}_{F A}
$$

The coupling term in the fluid weak form is similar and gives the transpose matrix multiplied by $\omega^{2}$.

\subsection{Discretized weak form}

By replacing the continuous pressure field as well as the structure displacements by their approximations in Equations (12) and (13), the following discretized system is obtained:

$$
\left(\left[\begin{array}{ccc}
\mathbf{K}_{\mathrm{FF}} & \mathbf{K}_{\mathrm{FA}} & \mathbf{0} \\
\mathbf{K}_{\mathrm{FA}}^{T} & \mathbf{K}_{\mathrm{AA}} & \mathbf{0} \\
\mathbf{0} & -\mathbf{C}_{\mathrm{SA}} & \mathbf{K}_{\mathrm{SS}}
\end{array}\right]-\omega^{2}\left[\begin{array}{ccc}
\mathbf{M}_{\mathrm{FF}} & \mathbf{M}_{\mathrm{FA}} & \mathbf{0} \\
\mathbf{M}_{\mathrm{FA}}^{T} & \mathbf{M}_{\mathrm{AA}} & \mathbf{C}_{\mathrm{SA}}^{T} \\
\mathbf{0} & \mathbf{0} & \mathbf{M}_{\mathrm{SS}}
\end{array}\right]\right)\left[\begin{array}{c}
\mathbf{P} \\
\mathbf{A} \\
\mathbf{U}_{\mathrm{S}}
\end{array}\right]=\left[\begin{array}{c}
\omega^{2} \mathbf{U}_{\mathrm{Fb}} \\
\mathbf{0} \\
\mathbf{F}_{\mathrm{S}}
\end{array}\right],
$$

where $\mathbf{K}_{\mathrm{SS}}$ and $\mathbf{M}_{\mathrm{SS}}$ are respectively the stiffness and mass matrices of the structure, $\mathbf{F}_{\mathrm{S}}$ is the discretized external load on the structure, $\mathbf{U}_{\mathrm{Fb}}$ is the discretized acoustic source, and the other matrices are defined as

$$
\begin{gathered}
\mathbf{K}_{F F}=\frac{1}{\rho_{f}} \int_{\Omega_{F}} \boldsymbol{B}_{F}^{T} \boldsymbol{B}_{F} \mathrm{~d} V, \\
\mathbf{M}_{F F}=\frac{1}{\rho_{f} c_{0}^{2}} \int_{\Omega_{F}} \boldsymbol{N}_{F}^{T} \boldsymbol{N}_{F} \mathrm{~d} V, \\
\mathbf{K}_{A A}=\boldsymbol{\beta}_{F A}^{T} \mathbf{K}_{F F} \boldsymbol{\beta}_{F A}, \\
\mathbf{K}_{F A}=\frac{1}{\rho_{f}} \int_{\Omega_{F}} H(\phi(M)) \boldsymbol{B}_{F}^{T} \boldsymbol{B}_{F} \mathrm{~d} V \boldsymbol{\beta}_{F A}, \\
\mathbf{M}_{F A}=\frac{1}{\rho_{f} c_{0}^{2}} \int_{\Omega_{F}} H(\phi(M)) \boldsymbol{N}_{F}^{T} \boldsymbol{N}_{F} \mathrm{~d} V \boldsymbol{\beta}_{F A}, \\
\mathbf{C}_{S A}=2 \int_{\Gamma} \boldsymbol{N}_{S}^{T} \mathbf{n}^{+} \boldsymbol{N}_{F} \mathrm{~d} \Sigma \boldsymbol{\beta}_{F A} .
\end{gathered}
$$

This nonsymmetric formulation can be made symmetric for a solving in the frequency domain by dividing the acoustic equations (first and second lines of the system) by $\omega^{2}(\omega \neq 0)$. 


\subsection{Integration of the enriched elements}

To compute the elemental enriched matrices $\mathbf{K}_{F A}$ and $\mathbf{M}_{F A}$, an analytical solution is used following the formulas given in [28] for a Heaviside enrichment combined with four-node tetrahedral elements.

Because $\boldsymbol{B}_{F}$ is constant in a four-node tetrahedral element, the elemental expression of $\mathbf{K}_{F A}$ in an enriched element $e$ becomes

$$
\mathbf{K}_{F A}^{e}=\frac{1}{\rho_{f}} \boldsymbol{B}_{F}^{T} \boldsymbol{B}_{F} \int_{\Omega_{F}^{e}} H(\phi(M)) \mathrm{d} V .
$$

The integral of the Heaviside function gives the following simple expression:

$$
\int_{\Omega_{F}^{e}} H(\phi(M)) \mathrm{d} V=\left(v_{e}^{+}-v_{e}^{-}\right) \frac{V_{e}}{v_{e}^{+}+v_{e}^{-}}=V_{e}\left(12 v_{e}^{+}-1\right),
$$

where the volumes of the positive and negative parent elemental domains are respectively $v_{e}^{+}$and $v_{e}^{-}$ (Figure 7) and $V_{e}$ is the total physical volume of the element. The expression is simplified because the total parent elemental volume is $v_{e}=v_{e}^{+}+v_{e}^{-}=1 / 6$.

The elemental expression of $\mathbf{M}_{F A}$ in an enriched element $e$ is

$$
\mathbf{M}_{F A}^{e}=\frac{1}{\rho_{f} c_{0}^{2}} \int_{\Omega_{F}^{e}} H(\phi(M)) N_{F}^{T} N_{F} \mathrm{~d} V .
$$

The integration is carried out by using two quadrature points in the parent element, one at the center of each domain noted $G^{+}$and $G^{-}$:

$$
\mathbf{M}_{F A}^{e}=\frac{1}{\rho_{f} c_{0}^{2}} V_{e}\left(\left.\left.6 v_{e}^{+} N_{F}^{T}\right|_{G^{+}} N_{F}\right|_{G^{+}}-\left.\left.\left(1-6 v_{e}^{+}\right) N_{F}^{T}\right|_{G^{-}} N_{F}\right|_{G^{-}}\right)
$$

A tetrahedron can be cut by the zero contour of the level-set in two ways as mentioned in Figure 7 in the parent element with coordinate system $(r, s, t)$ : case 1-3 where one node is one on side and three nodes are on the other side; case 2-2 where two nodes are on each side. Ventura [28] gives only the formulas for the first case (1-3), whereas nothing is mentioned for the second case (2-2). The analytical formulas are recalled for the first case and developed for the second in the following.

The first case is supposed to have node 4 in domain $\Omega^{+}$where $\phi>0$ and nodes 1,2 and 3 in domain $\Omega^{-}$where $\phi<0$. The opposite situation according to the signed of $\phi$ is obtained by switching $v_{e}^{+}$and $v_{e}^{-}$. With the notations defined in Figure 7(a), the parent volume of the positive side $v_{e}^{+}$is simply the volume of a subtetrahedron domain bounded by the four following points:

$$
(0,0,0),\left(r_{0}, 0,0\right),\left(0, s_{0}, 0\right),\left(0,0, t_{0}\right),
$$

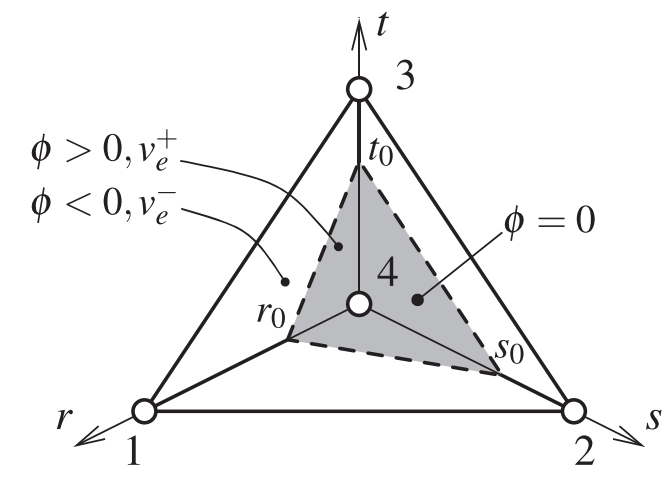

(a) Case 1-3

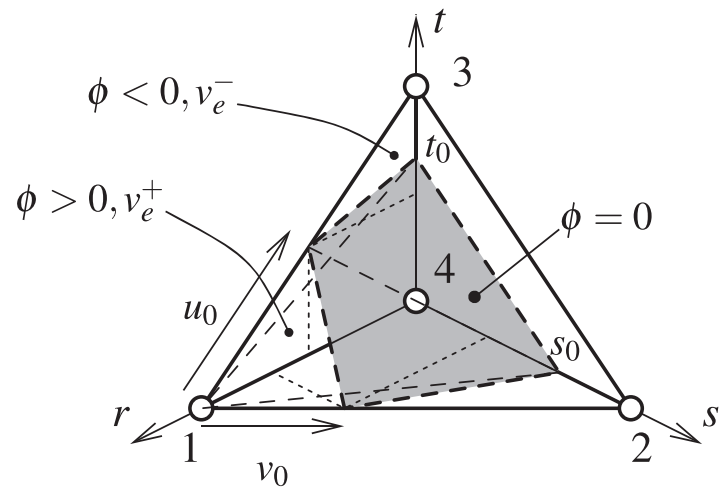

(b) Case 2-2

Figure 7. Tetrahedral finite element in the parent domain cut by the zero contour of the level-set. (a) Case 1-3; (b) case 2-2. 
Table I. Subdecomposition of the positive domain for case 2-2 into three subtetrahedra.

\begin{tabular}{ll}
\hline Four tetrahedron vertices & Tetrahedron volume \\
\hline$(0,0,0),(1,0,0),\left(0, s_{0}, 0\right),\left(0,0, t_{0}\right)$ & $v_{1}=\frac{s_{0} t_{0}}{6}$ \\
$(1,0,0),\left(0, s_{0}, 0\right),\left(0,0, t_{0}\right),\left(1-\frac{\sqrt{2}}{2} u_{0}, 0, \frac{\sqrt{2}}{2} u_{0}\right)$ & $v_{2}=\frac{\sqrt{2}}{12} s_{0} u_{0}\left(1-t_{0}\right)$ \\
$(1,0,0),\left(0, s_{0}, 0\right),\left(1-\frac{\sqrt{2}}{2} u_{0}, 0, \frac{\sqrt{2}}{2} u_{0}\right),\left(1-\frac{\sqrt{2}}{2} v_{0}, \frac{\sqrt{2}}{2} v_{0}, 0\right)$ & $v_{3}=\frac{u_{0} v_{0}\left(1-s_{0}\right)}{12}$ \\
\hline
\end{tabular}

where $r_{0}, s_{0}$, and $t_{0}$ are defined such that $\phi\left(r_{0}, 0,0\right)=\phi\left(0, s_{0}, 0\right)=\phi\left(0,0, t_{0}\right)=0$. For this situation, as mentioned in [28], the $v_{e}^{+}$volume is

$$
v_{e}^{+}=\frac{r_{0} s_{0} t_{0}}{6}
$$

Coordinates in the parent element of the center points $G^{+}$and $G^{-}$are given by

$$
G^{+}:\left(\frac{r_{0}}{4}, \frac{s_{0}}{4}, \frac{t_{0}}{4}\right) \text { and } G^{-}:\left(\frac{1-r_{0}^{2} s_{0} t_{0}}{4\left(1-r_{0} s_{0} t_{0}\right)}, \frac{1-r_{0} s_{0}^{2} t_{0}}{4\left(1-r_{0} s_{0} t_{0}\right)}, \frac{1-r_{0} s_{0} t_{0}^{2}}{4\left(1-r_{0} s_{0} t_{0}\right)}\right)
$$

The second case is supposed to have nodes 1 and 4 in domain $\Omega^{+}$where $\phi>0$ and nodes 2 and 3 in domain $\Omega^{-}$where $\phi<0$. With the notations defined in Figure 7(a), the parent volume of the positive side $v_{e}^{+}$is the volume of three assembled subtetrahedra domains. The two variables $s_{0}$ and $t_{0}$ go from 0 to 1 and are defined such that $\phi\left(0, s_{0}, 0\right)=\phi\left(0,0, t_{0}\right)=0$. The two variables $u_{0}$ and $v_{0}$ go from 0 to $\sqrt{2}$ and are defined such that $\phi\left(1-\sqrt{2} / 2 u_{0}, 0, \sqrt{2} / 2 u_{0}\right)=$ $\phi\left(1-\sqrt{2} / 2 v_{0}, \sqrt{2} / 2 v_{0}, 0\right)=0$. The coordinates of the four vertices of each tetrahedron as well as their volumes are given in Table I.

The parent volume of the positive side $v_{e}^{+}$is given by

$$
v_{e}^{+}=v_{1}+v_{2}+v_{3}=\frac{1}{12}\left(2 s_{0} t_{0}+\sqrt{2} s_{0} u_{0}-\sqrt{2} s_{0} u_{0} t_{0}+u_{0} v_{0}-u_{0} v_{0} s_{0}\right) .
$$

The center points $G^{+}$and $G^{-}$are given by their coordinate vectors using the following barycentric equations:

$$
\begin{gathered}
\boldsymbol{O} \boldsymbol{G}^{+}=\frac{1}{v_{e}^{+}}\left(v_{1} \boldsymbol{O} \boldsymbol{G}_{1}+v_{2} \boldsymbol{O} \boldsymbol{G}_{2}+v_{3} \boldsymbol{O} \boldsymbol{G}_{3}\right), \\
\boldsymbol{O} \boldsymbol{G}^{-}=\frac{1}{1-6 v_{e}^{+}}\left(\boldsymbol{O G}-6 v_{e}^{+} \boldsymbol{O} \boldsymbol{G}^{+}\right),
\end{gathered}
$$

where $\boldsymbol{O} \boldsymbol{G}_{i}$ denotes the coordinate vector of the center of the subtetrahedron number $i$ assuming that point $O$ is localized at $(0,0,0)$.

\section{STRATEGY FOR A PARAMETRIC STUDY OF SEVERAL IMMERSED STRUCTURES}

The discretized system to be solved for a configuration of the coupled fluid-structure system is given by Equation (28). This system can be extended to $n$ immersed structures as

$$
\left(K-w^{2} M\right) \mathcal{X}=F
$$


with

$$
\begin{aligned}
& \mathscr{K}=\left[\begin{array}{cccccccc}
\mathbf{K}_{\mathrm{FF}} & \mathbf{K}_{\mathrm{FA}_{1}} & \mathbf{0} & \mathbf{K}_{\mathrm{FA}_{2}} & \mathbf{0} & \vdots & \mathbf{K}_{\mathrm{FA}_{n}} & \mathbf{0} \\
\mathbf{K}_{\mathrm{FA}}^{T} & \mathbf{K}_{\mathrm{A}_{1} \mathrm{~A}_{1}} & \mathbf{0} & \mathbf{0} & \mathbf{0} & \vdots & \mathbf{0} & \mathbf{0} \\
\mathbf{0} & -\mathbf{C}_{\mathrm{S}_{1} \mathrm{~A}_{1}} & \mathbf{K}_{\mathrm{S}_{1} \mathrm{~S}_{1}} & \mathbf{0} & \mathbf{0} & \vdots & \mathbf{0} & \mathbf{0} \\
\mathbf{K}_{\mathrm{FA}_{2}}^{T} & \mathbf{0} & \mathbf{0} & \mathbf{K}_{\mathrm{A}_{2} \mathrm{~A}_{2}} & \mathbf{0} & \vdots & \mathbf{0} & \mathbf{0} \\
\mathbf{0} & \mathbf{0} & \mathbf{0} & -\mathbf{C}_{\mathrm{S}_{2} \mathrm{~A}_{2}} & \mathbf{K}_{\mathrm{S}_{2} \mathrm{~S}_{2}} & \vdots & \mathbf{0} & \mathbf{0} \\
\cdots & \cdots & \cdots & \cdots & \cdots & \cdots & \cdots & \cdots \\
\mathbf{K}_{\mathrm{FA}}^{T} & \mathbf{0} & \mathbf{0} & \mathbf{0} & \mathbf{0} & \vdots & \mathbf{K}_{\mathrm{A}_{n} \mathrm{~A}_{n}} & \mathbf{0} \\
\mathbf{0} & \mathbf{0} & \mathbf{0} & \mathbf{0} & \mathbf{0} & \vdots & -\mathbf{C}_{\mathrm{S}_{n} \mathrm{~A}_{n}} & \mathbf{K}_{\mathrm{S}_{n} \mathrm{~S}_{n}}
\end{array}\right], \\
& \mathscr{M}=\left[\begin{array}{cccccccc}
\mathbf{M}_{\mathrm{FF}} & \mathbf{M}_{\mathrm{FA}_{1}} & \mathbf{0} & \mathbf{M}_{\mathrm{FA}_{2}} & \mathbf{0} & \vdots & \mathbf{M}_{\mathrm{FA}_{\mathrm{n}}} & \mathbf{0} \\
\mathbf{M}_{\mathrm{FA}}^{T} & \mathbf{M}_{\mathrm{A}_{1} \mathrm{~A}_{1}} & \mathbf{C}_{\mathrm{S}_{1} \mathrm{~A}_{1}}^{T} & \mathbf{0} & \mathbf{0} & \vdots & \mathbf{0} & \mathbf{0} \\
\mathbf{0} & \mathbf{0} & \mathbf{M}_{\mathrm{S}_{1} \mathrm{~S}_{1}} & \mathbf{0} & \mathbf{0} & \vdots & \mathbf{0} & \mathbf{0} \\
\mathbf{M}_{\mathrm{FA}_{2}}^{T} & \mathbf{0} & \mathbf{0} & \mathbf{M}_{\mathrm{A}_{2} \mathrm{~A}_{2}} & \mathbf{C}_{\mathrm{S}_{2} \mathrm{~A}_{2}}^{T} & \vdots & \mathbf{0} & \mathbf{0} \\
\mathbf{0} & \mathbf{0} & \mathbf{0} & \mathbf{0} & \mathbf{M}_{\mathrm{S}_{2} \mathrm{~S}_{2}} & \vdots & \mathbf{0} & \mathbf{0} \\
\cdots & \cdots & \cdots & \cdots & \cdots & \cdots & \cdots & \cdots \\
\mathbf{M}_{\mathrm{FA}}^{T} & \mathbf{0} & \mathbf{0} & \mathbf{0} & \mathbf{0} & \vdots & \mathbf{M}_{\mathrm{A}_{\mathrm{n}} \mathrm{A}_{\mathrm{n}}} & \mathbf{C}_{\mathrm{S}_{\mathrm{n}} \mathrm{A}_{\mathrm{n}}}^{T} \\
\mathbf{0} & \mathbf{0} & \mathbf{0} & \mathbf{0} & \mathbf{0} & \vdots & \mathbf{0} & \mathbf{M}_{\mathrm{S}_{\mathrm{n}} \mathrm{S}_{\mathrm{n}}}
\end{array}\right] \\
& \mathcal{X}=\left[\begin{array}{c}
\mathbf{P} \\
\mathbf{A}_{1} \\
\mathbf{U}_{\mathrm{S} 1} \\
\mathbf{A}_{2} \\
\mathbf{U}_{\mathrm{S} 2} \\
\vdots \\
\mathbf{A}_{n} \\
\mathbf{U}_{\mathrm{S} n}
\end{array}\right], \mathcal{F}=\left[\begin{array}{c}
\omega^{2} \mathbf{U}_{\mathrm{Fb}} \\
\mathbf{0} \\
\mathbf{F}_{\mathrm{S}_{1}} \\
\mathbf{0} \\
\mathbf{F}_{\mathrm{S}_{2}} \\
\vdots \\
\mathbf{0} \\
\mathbf{F}_{\mathrm{S}_{\mathrm{n}}}
\end{array}\right]
\end{aligned}
$$

where subscripts 1 to $n$ denote the $n$ different structures. It is supposed in this work that the structures are far enough from each other to not share enriched nodes. With this assumption, only one level-set can be used to localize several structures in the fluid domain.

In system (44), matrices $\mathbf{K}_{\mathrm{FF}}$ and $\mathbf{M}_{\mathrm{FF}}$ are those corresponding to the empty fluid cavity, meaning that they do not need to be recomputed when the structures are placed differently. Matrices $\mathbf{K}_{\mathrm{A}_{\mathrm{i}} \mathrm{A}_{\mathrm{i}}}$ and $\mathbf{M}_{\mathrm{A}_{\mathrm{i}} \mathrm{A}_{\mathrm{i}}}$ do not need to be recomputed because they can be computed from $\mathbf{K}_{\mathrm{FF}}$ and $\mathbf{M}_{\mathrm{FF}}$ by using the localization matrix $\boldsymbol{\beta}_{F A_{i}}$ (Equations 31 and 32). Matrices $\mathbf{K}_{\mathrm{S}_{\mathrm{i}} \mathrm{S}_{\mathrm{i}}}$ and $\mathbf{M}_{\mathrm{S}_{\mathrm{i}} \mathrm{S}_{\mathrm{i}}}$ are those corresponding to the structures with no fluid; again, they do not need to be recomputed for each configuration unless the geometry of the structure itself is changed. Finally, when many structures are placed arbitrarily in the fluid, the only matrices to be computed in the parametric study are the coupling matrices between the standard part and the enrichment of the pressure $\left(\mathbf{K}_{\mathrm{FA}_{i}}, \mathbf{M}_{\mathrm{FA}_{\mathrm{i}}}\right)$ and the coupling matrices between the fluid enrichment and the structures $\mathbf{C}_{\mathrm{S}_{\mathrm{i}} \mathrm{A}_{\mathrm{i}}}$.

The algorithm used to compute frequency response functions during a parametric study is

- Compute $\mathbf{K}_{\mathrm{FF}}$ and $\mathbf{M}_{\mathrm{FF}}$ from the given empty cavity mesh

- Compute $\mathbf{K}_{\mathrm{S}_{\mathrm{i}} \mathrm{S}_{\mathrm{i}}}$ and $\mathbf{M}_{\mathrm{Si}_{\mathrm{i}} \mathrm{S}_{\mathrm{i}}}$ for each structure

- Loop over the parameters: 
- Loop over the structures that have been modified:

- Update structure position.

- If the structure geometry has changed, compute $\mathbf{K}_{\mathrm{S}_{\mathrm{i}} \mathrm{S}_{\mathrm{i}}}$ and $\mathbf{M}_{\mathrm{S}_{\mathrm{i}} \mathrm{S}_{\mathrm{i}}}$.

- Compute the level-set $\phi(M)$.

- Compute $\boldsymbol{\beta}_{F A_{i}}$ matrix; in other words, get the set of enriched nodes $\mathscr{A}_{i}$.

- Compute the fluid-enrichment coupling matrices $\mathbf{K}_{\mathrm{FA}_{\mathrm{i}}}$ and $\mathbf{M}_{\mathrm{FA}_{\mathrm{i}}}$.

- Compute the structure-enrichment coupling matrix $\mathbf{C}_{\mathrm{S}_{\mathrm{i}} \mathrm{A}_{\mathrm{i}}}$.

- Form the system as in Equation (44).

- Compute the frequency response function for this configuration.

The algorithm could be simplified by considering all structures as one, avoiding the structure loop. However, it may become difficult to modify one specific structure.

The implementation has been carried out with Python for the main program at the global level and using Fortran functions at the elemental level. This Python-Fortran coupling takes benefit of the two different languages: speed of Fortran compiled functions and ease of Python to manipulate large sparse matrices. Geometry, meshes, and results viewing are performed with Gmsh [29]. This implementation strategy allows to deal with quite large number of degrees of freedom.

\section{VALIDATION AND APPLICATIONS}

\subsection{Validation on a parallelipipedic cavity}

A parallelipipedic cavity divided into two parts by a plane flexible structure is considered (Figure 8). The data of the problem are given in Table II. The structure is simply supported on its four edges; a harmonic load of amplitude $1 \mathrm{~N}$ is applied to point $A$ in the normal direction of the plane structure. The position of point $A$ is chosen such that it cannot be a vibration node of the structure (Figure 8). The studied frequency range is 10 to $600 \mathrm{~Hz}$.

5.1.1. Analysis of the problem. Because the air is a light fluid compared with the structure, the modes are almost decoupled; therefore, a separate modal analysis of the fluid and the structure is carried out to verify the convergence of the numerical results obtained by the coupled system.

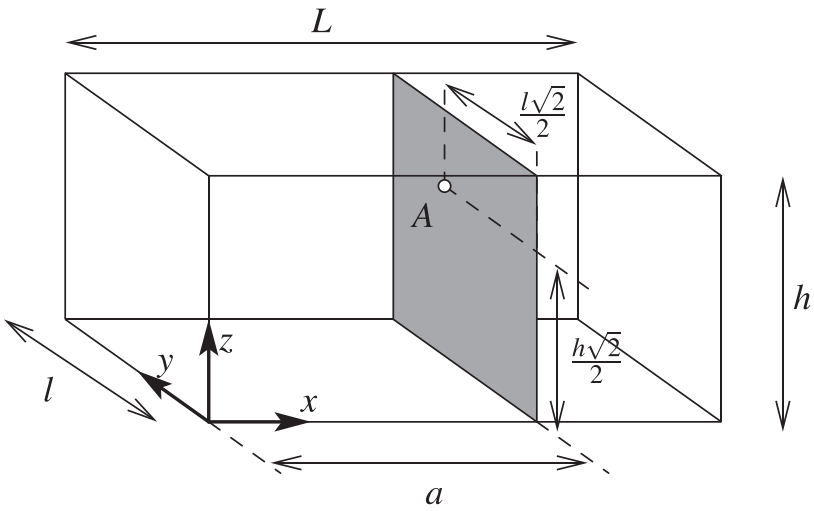

Figure 8. Parallelipipedic cavity divided into two parts by a flexible structure.

Table II. Data of the parallelipipedic cavity cut by a plane structure.

\begin{tabular}{llcccc}
\hline \multicolumn{2}{c}{ Geometry } & \multicolumn{2}{c}{ Fluid } & \multicolumn{2}{c}{ Structure } \\
\hline$L(\mathrm{~m})$ & 1 & $c_{0}\left(\mathrm{~m} \cdot \mathrm{s}^{-1}\right)$ & 340 & $E(\mathrm{~Pa})$ & $70,000 \times 10^{6}$ \\
$l(\mathrm{~m})$ & 0.6 & $\rho_{F}\left(\mathrm{~kg} \cdot \mathrm{m}^{-3}\right)$ & 1.2 & $v$ & 0.27 \\
$h(\mathrm{~m})$ & 0.4 & & & $\rho_{S}\left(\mathrm{~kg} \cdot \mathrm{m}^{-3}\right)$ & 2700 \\
$a(\mathrm{~m})$ & 0.75 & & & $e$, thickness $(\mathrm{m})$ & $4 \times 10^{-3}$ \\
\hline
\end{tabular}


Table III. Eigenfrequencies of the two parallelipipedic fluid cavities.

\begin{tabular}{lccl}
\hline$i$ & $j$ & $k$ & $f(\mathrm{~Hz})$ \\
\hline \multicolumn{2}{l}{ Large } & cavity, & $L_{x}=a$ \\
1 & 0 & 0 & \\
0 & 1 & 0 & 226.7 \\
1 & 1 & 0 & 363.3 \\
0 & 0 & 1 & 325 \\
2 & 0 & 0 & 353.3 \\
1 & 0 & 1 & 381.7 \\
0 & 1 & 1 & 510.8 \\
2 & 1 & 0 & 533.6 \\
1 & 1 & 1 & 558.8 \\
0 & 2 & 0 & 566.7 \\
& & & \\
Small cavity, $L_{x}=L-a$ & \\
0 & 1 & 0 & 283.3 \\
0 & 0 & 1 & 325 \\
0 & 1 & 1 & 510.8 \\
0 & 2 & 0 & 566.7 \\
\hline
\end{tabular}

In the case of a rigid structure, the two cavities are decoupled. The analytical eigenmodes $p_{[i j k]}$ and eigenfrequencies $f_{[i j k]}$ of the fluid cavities are given by

$$
\begin{gathered}
p_{[i j k]}(x, y, z)=\cos \frac{i \pi x}{L_{x}} \cos \frac{j \pi y}{l} \cos \frac{k \pi z}{h}, \\
f_{[i j k]}=\frac{c}{2}\left(\frac{i^{2}}{L_{x}^{2}}+\frac{j^{2}}{l^{2}}+\frac{k^{2}}{h^{2}}\right)^{\frac{1}{2}},
\end{gathered}
$$

where $i, j$, and $k$ are integers and $L_{x}$ is the length of the cavity along $x$ direction $\left(L_{x}=a\right.$ for the large cavity and $L_{x}=L-a$ for the small one). Table III gives the eigenfrequencies of the two cavities up to $600 \mathrm{~Hz}$.

The analytical eigenmodes and eigenfrequencies of the four-edge simply supported structure are given by

$$
\begin{gathered}
w_{[i j]}(x, y)=\sin \frac{i \pi y}{l} \sin \frac{j \pi z}{h}, \\
f_{[i j]}=\sqrt{\frac{E e^{2}}{12\left(1-v^{2}\right) \rho_{S}}}\left(\frac{i^{2} 2}{l^{2}}+\frac{j^{2} 2}{h^{2}}\right),
\end{gathered}
$$

where $i$ and $j$ are integers and $w_{[i j]}$ is the transverse displacement. Table IV gives the analytical eigenfrequencies of the structure up to $600 \mathrm{~Hz}$.

5.1.2. Frequency analysis. The frequency response function is computed using the present approach based on XFEM as well as using a conforming mesh between fluid and structure at the interface to validate the results. The two meshes are presented in Figure 9. Table V shows the number of nodes and elements used for each computation. The number of enriched nodes (set of nodes $\mathscr{A}$ ) is 1129 , and there are 3067 enriched elements for the XFEM computation. The sound level indicator is the mean quadrature pressure, expressed in $\mathrm{dB}$, and computed according to the following definition:

$$
L_{p}=10 \log _{10} \frac{\int_{\Omega_{\mathrm{F}}} p^{2} \mathrm{~d} V}{p_{0}^{2} \int_{\Omega_{\mathrm{F}}} \mathrm{d} V},
$$

where $p_{0}=20 \mu \mathrm{Pa}$ is the reference sound pressure in the air. 
Table IV. Eigenfrequencies of the plane structure.

\begin{tabular}{llc}
\hline$i$ & $j$ & $f(\mathrm{~Hz})$ \\
\hline 1 & 1 & 86.6 \\
2 & 1 & 166.5 \\
1 & 2 & 266.3 \\
3 & 1 & 299.7 \\
2 & 2 & 336.3 \\
3 & 2 & 379.6 \\
3 & 1 & 386.2 \\
1 & 3 & 566.2 \\
\hline
\end{tabular}
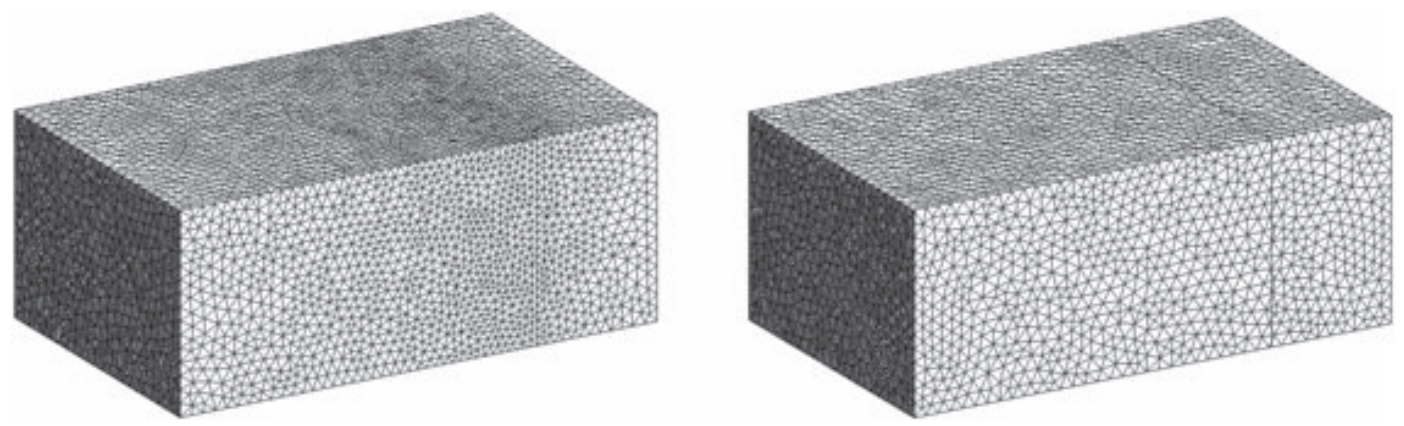

Figure 9. The two meshes of the parallelipipedic cavity: left, compatible; right, noncompatible for XFEM.

Table V. Mesh characteristics of the parallelipipedic cavity.

\begin{tabular}{|c|c|c|c|c|c|c|c|}
\hline \multicolumn{4}{|c|}{ Compatible mesh } & \multicolumn{4}{|c|}{ XFEM mesh } \\
\hline \multicolumn{2}{|c|}{ Fluid mesh } & \multicolumn{2}{|c|}{ Structure mesh } & \multicolumn{2}{|c|}{ Fluid mesh } & \multicolumn{2}{|c|}{ Structure mesh } \\
\hline Nodes & Elements & Nodes & Elements & Nodes & Elements & Nodes & Elements \\
\hline 44,643 & 263,274 & 1232 & 2334 & 21,089 & 120,306 & 1243 & 2356 \\
\hline
\end{tabular}

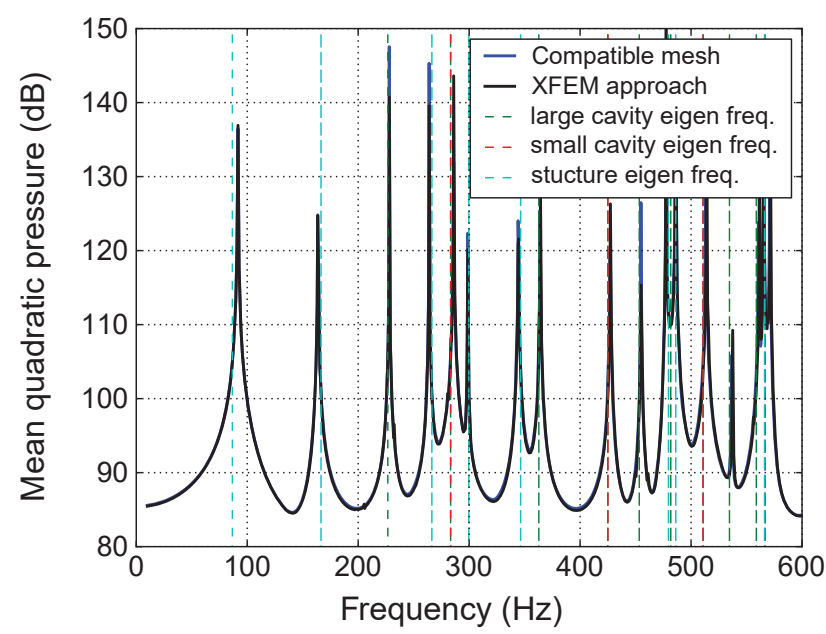

Figure 10. Frequency response function of the parallelipipedic cavity separated by a plane flexible structure.

The comparison of the frequency response functions is shown in Figure 10. On this figure, the decoupled analytical eigenfrequencies of the two cavities as well as those of the structure are mentioned as vertical lines. The coupled problem eigenfrequencies corresponding to the resonances of the FRF are close to these vertical lines. The results are compared for four frequencies close to the four first resonances of the frequency response function in Figure 11; the structure is placed above the fluid cavity to visualize it. The XFEM results show a discontinuous jump at the interface because the plot routine does not take into account the Heaviside enrichment in an element, 

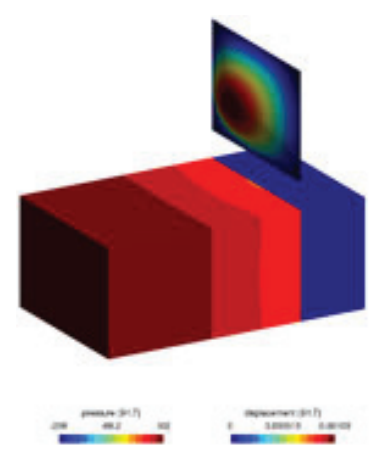

(a) Reference, $f=92 \mathrm{~Hz}$
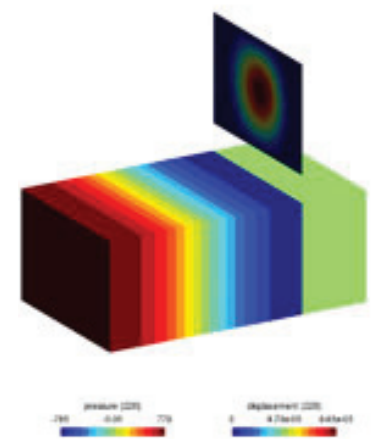

(e) Reference, $f=228 \mathrm{~Hz}$
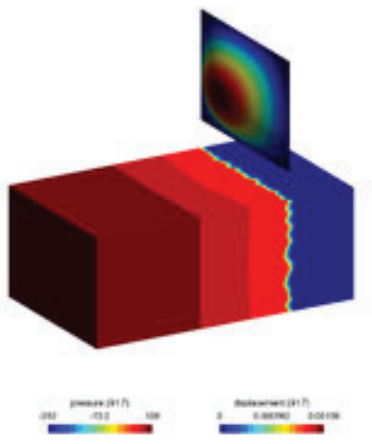

(b) XFEM, $f=92 \mathrm{~Hz}$
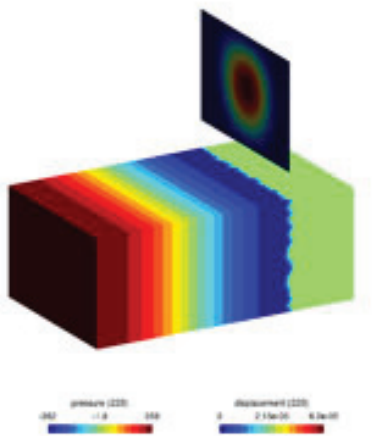

(f) XFEM, $f=228 \mathrm{~Hz}$
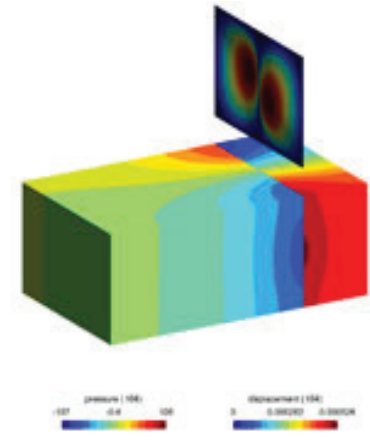

(c) Reference, $f=164 \mathrm{~Hz}$
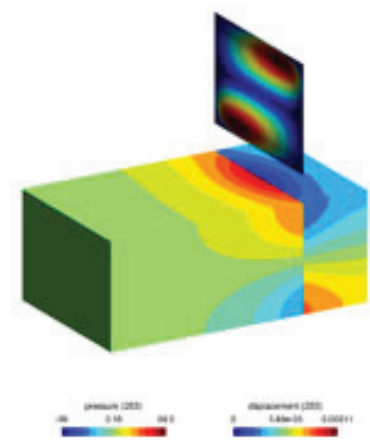

(g) Reference, $f=263 \mathrm{~Hz}$
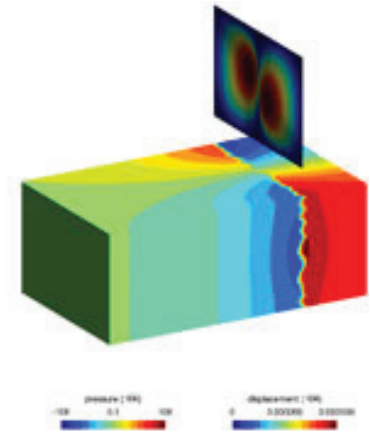

(d) XFEM, $f=164 \mathrm{~Hz}$
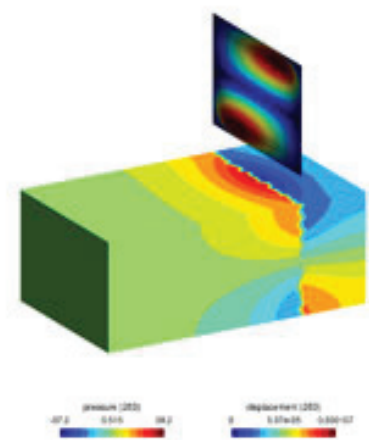

(h) XFEM, $f=263 \mathrm{~Hz}$

Figure 11. Results at different frequencies close to resonances of the coupled system. (a) Reference, $f=92 \mathrm{~Hz}$; (b) XFEM, $f=92 \mathrm{~Hz}$; (c) Reference, $f=164 \mathrm{~Hz}$; (d) XFEM, $f=164 \mathrm{~Hz}$; (e) Reference, $f=228 \mathrm{~Hz}$; (f) XFEM, $f=228 \mathrm{~Hz}$; (g) Reference, $f=263 \mathrm{~Hz}$; (h) XFEM, $f=263 \mathrm{~Hz}$.

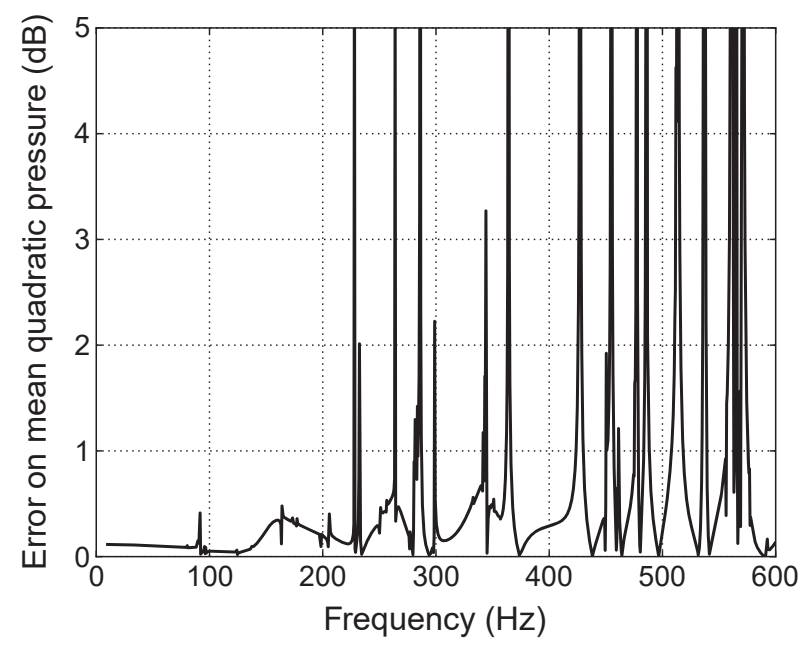

Figure 12. dB difference between the compatible and the XFEM results for the parallelipipedic cavity.

whereas the enrichment is used to compute the real pressure at an enriched node according to Equation (22). The plots show the good ability of the method to correctly catch the pressure jump through the structure.

$\mathrm{A} \mathrm{dB}$ difference error between the two results obtained by the two methods is computed in Figure 12. This error is more a qualitative tool than a quantitative tool for two main reasons:

- The compatible mesh has been made smaller to have a good reference solution, so the error contains the discretization error as well as an error coming from the XFEM method itself.

- There is no damping in this problem, so the solution becomes highly singular at resonances; the value has thus no signification close to a resonance. 
For these reasons, the error is not a relative error (it would add more uncertainty in the value), and the error is arbitrarily cut up to a value of $5 \mathrm{~dB}$. However, the difference is less than $1 \mathrm{~dB}$ up to $225 \mathrm{~Hz}$ and globally increases to less than $2 \mathrm{~dB}$ away from the resonances, which represents approximately $2 \%$ in terms of relative error.

5.1.3. Parametric study. To show the ability of the proposed approach to perform a parametric study according to the structure position, the plane flexible structure is arbitrarily placed at four different positions ( $a=0.5, a=0.55, a=0.65$, and $a=0.75$ ), whereas the fluid mesh remains to be the same. The fluid and structure meshes used for this parametric study are the same as those used in the previous section for the XFEM noncompatible case. The number of enriched nodes vary from one configuration to an other because the zero-contour level-set is placed differently in the fluid mesh: 1062 enriched nodes for $a=0.5,1047$ enriched nodes for $a=0.55,1082$ enriched nodes for $a=0.65$, and 1129 enriched nodes for $a=0.75$. The different frequency response functions are plotted in Figure 13. Because the first two resonances correspond to structure eigenfrequencies, these are not shifted whereas all the cavity resonances are shifted according to the $a$ value. The limit case $a=0.5$ is where the two cavities have exactly the same size, meaning that their eigenfrequencies overlap.

5.1.4. Validation of the proposed approach. This application shows that the method can reproduce a pressure discontinuity in a noncompatible mesh. The $\mathrm{dB}$ difference error between a reference solution and the XFEM approach is small, even if the mesh used with the XFEM method contains less elements. A parametric study of the position of a structure in the fixed fluid mesh of the empty cavity is easy to perform and gives accurate results.

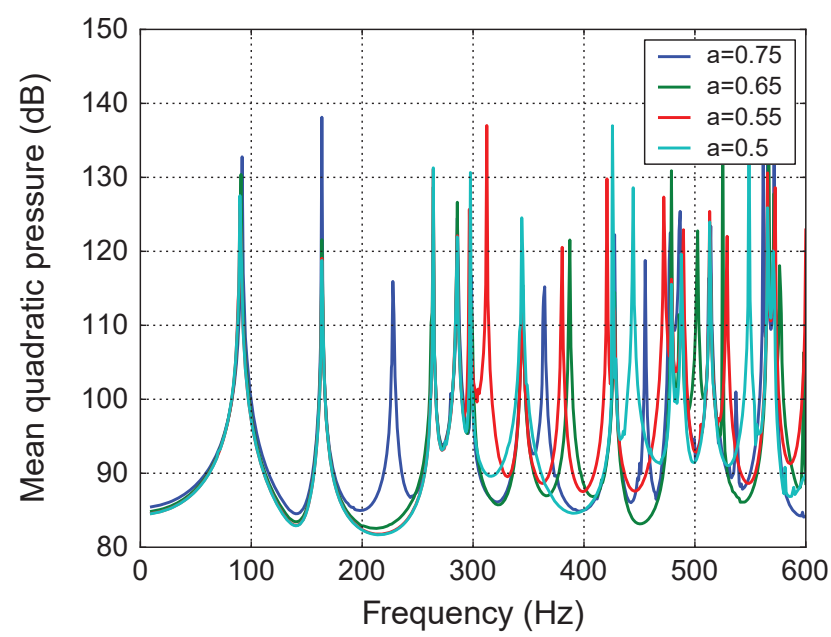

Figure 13. Parametric study results for the parallelipipedic cavity separated by a structure.

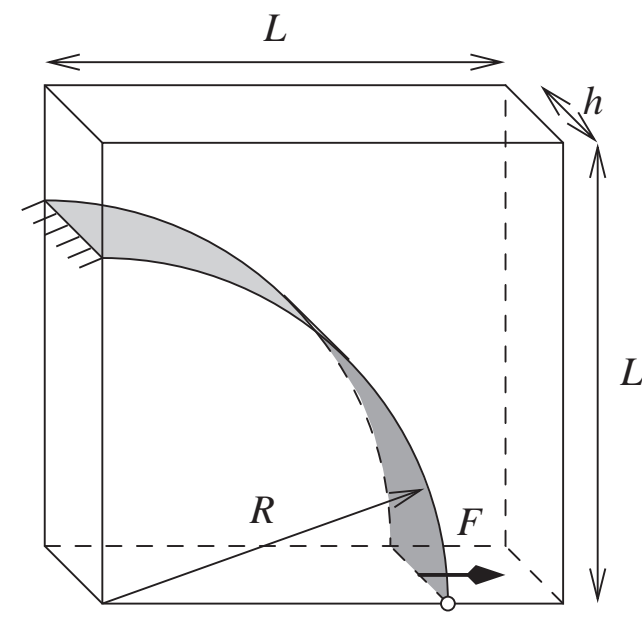

Figure 14. One-fourth of a circular structure ring immersed in a thin cavity. 
Table VI. Data of the circular structure ring immersed in a thin cavity.

\begin{tabular}{lccc}
\hline \multicolumn{3}{c}{ Fluid } & \multicolumn{2}{c}{ Structure } \\
\hline$L(\mathrm{~m})$ & 1 & $E(\mathrm{~Pa})$ & $70,000 \times 10^{6}$ \\
$h(\mathrm{~m})$ & 0.03 & $v$ & 0.27 \\
& & $\rho_{S}\left(\mathrm{~kg} \cdot \mathrm{m}^{-3}\right)$ & 2700 \\
$c_{0}\left(\mathrm{~m} \cdot \mathrm{s}^{-1}\right)$ & 340 & $e$, thickness $(\mathrm{m})$ & $6 \times 10^{-3}$ \\
$\rho_{F}\left(\mathrm{~kg} \cdot \mathrm{m}^{-3}\right)$ & 1.2 & $R$, radius $(\mathrm{m})$ & 0.8 \\
& & $F$, harmonic load $(\mathrm{N})$ & 1 \\
\hline
\end{tabular}

Table VII. Parameters and results of the convergence study for the immersed circular ring.

\begin{tabular}{lccccc}
\hline Mesh & $\mathrm{a}$ & $\mathrm{b}$ & $\mathrm{c}$ & $\mathrm{d}$ & $\mathrm{e}$ \\
\hline Fluid elements & 216 & 726 & 2646 & 10,086 & 196,830 \\
Fluid nodes & 98 & 288 & 968 & 3528 & 40,344 \\
Element length & 0.16 & 0.09 & 0.05 & 0.025 & 0.012 \\
Mean dB difference & 7.73 & 3.41 & 1.37 & 0.45 & 0.21 \\
\hline
\end{tabular}

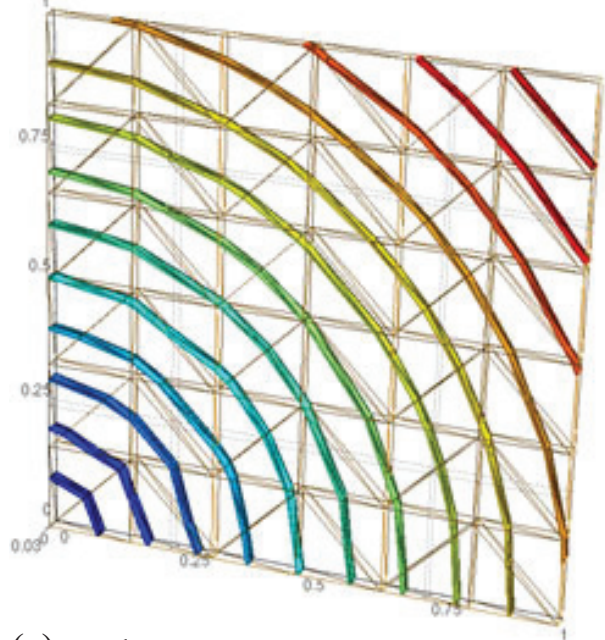

(a) Mesh $a$

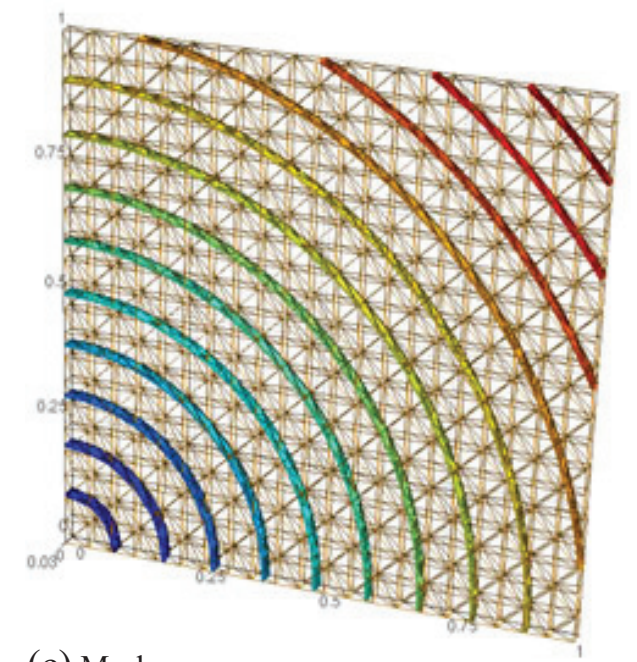

(c) Mesh $c$

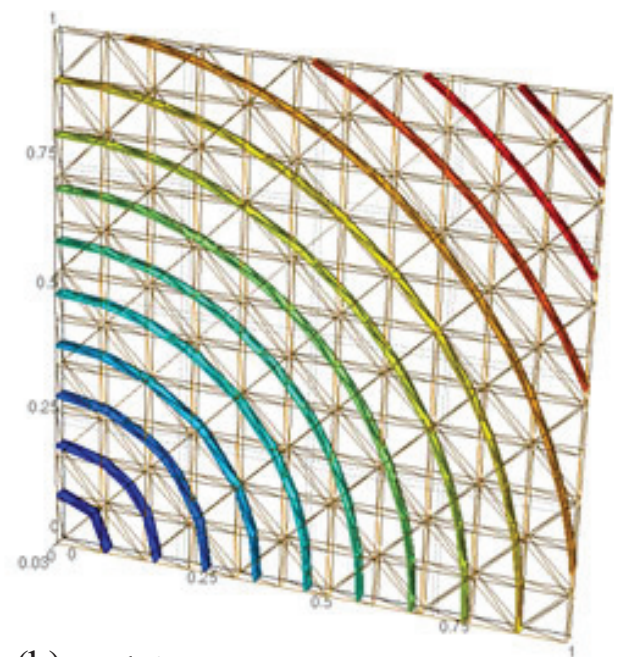

(b) Mesh $b$

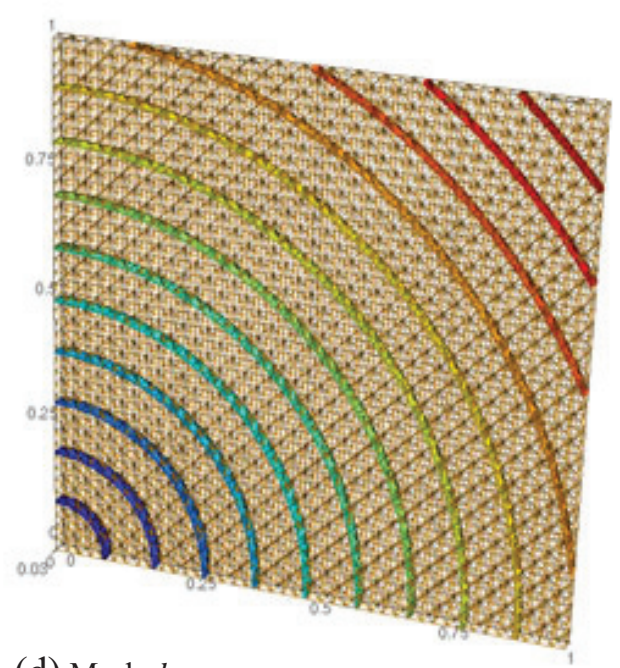

(d) Mesh $d$

Figure 15. Iso-contours of the level-set for the immersed circular ring. (a) Mesh a; (b) mesh b; (c) mesh c; (d) mesh d. 


\subsection{Convergence study on an immersed circular ring structure}

The accuracy of the method is principally based on the interface discretization. This discretization depends on the fluid element length compared with the structure curvature. To study this effect on the convergence, one-fourth of a circular ring structure is immersed in a thin square cavity of thickness denoted by $h$ (Figure 14 and Table VI). Boundary conditions as well as load are chosen such that the solution of the problem is two dimensional.

One side of the structure is clamped; a harmonic load of amplitude $1 \mathrm{~N}$ is applied on the other side. A reference solution is obtained by using a compatible mesh: 1018 nodes and 1676 elements for the structure; 123,748 nodes and 631,012 elements for the fluid.

The structure mesh is chosen to be the same as for the reference solution. Five different structured fluid meshes are used; their characteristics are shown in Table VII. The fluid mesh as well as the level-set projections are shown for the first four meshes in Figure 15. It can be visually observed that meshes $a$ and $b$ gives a quite inaccurate representation of the level-set, specially around the circle center. The enriched elements are plotted in Figure 16 for the first four meshes: meshes $a$ and $b$ have large partition of unity supports around the interface, leading to a poor approximation of the Heaviside function enrichment.

The frequency response function is computed for these five fluid meshes and are compared with the reference solution (Figure 17(a) and 17(b)). The frequency response function for mesh $e$ is not plotted because the difference with the reference solution cannot be observed visually. A dB difference with the reference solution is computed for each mesh and for each frequency. This $\mathrm{dB}$ difference is plotted in Figure 17(c) for meshes $c$ and $e$ because it is too large for meshes $a$ and $b$;

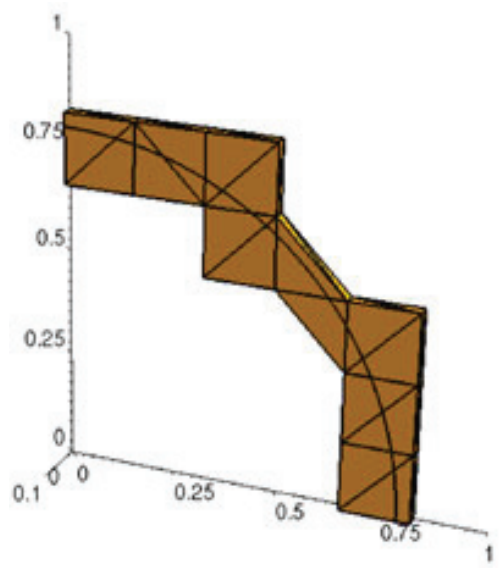

(a) Mesh $a$

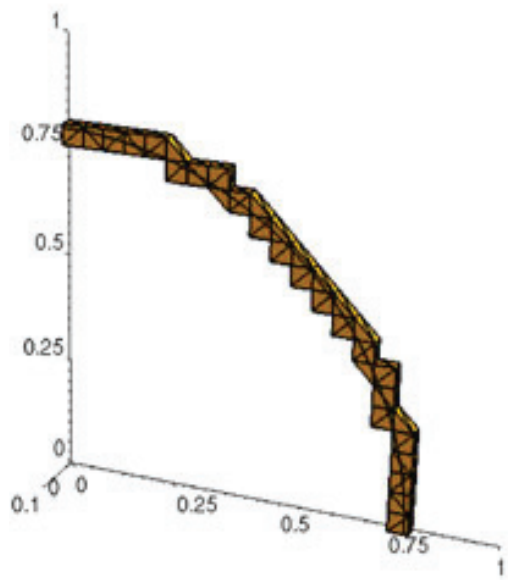

(c) Mesh c

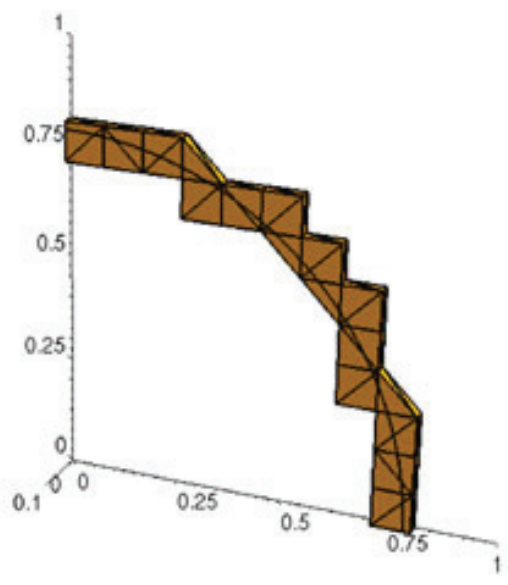

(b) Mesh $b$

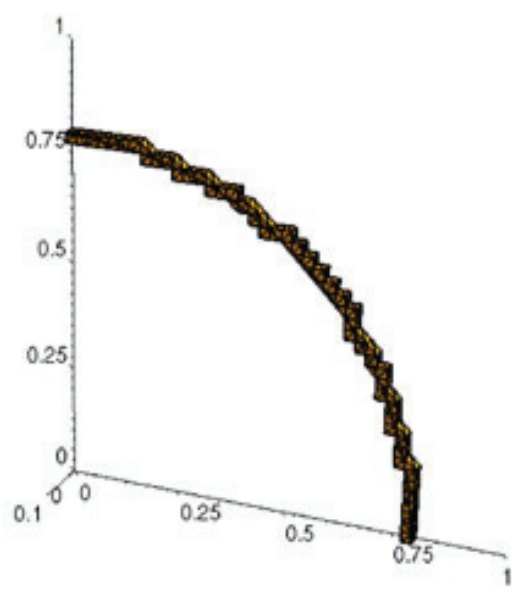

(d) Mesh $d$

Figure 16. Enriched elements for the immersed circular ring. (a) Mesh a; (b) mesh b; (c) mesh c; (d) mesh d. 


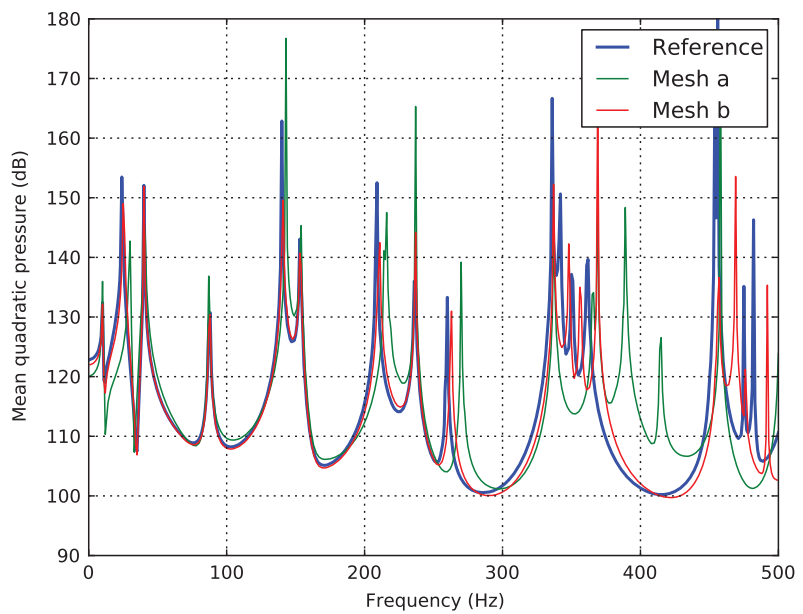

(a) Frequency response function, mesh $a$ and $b$

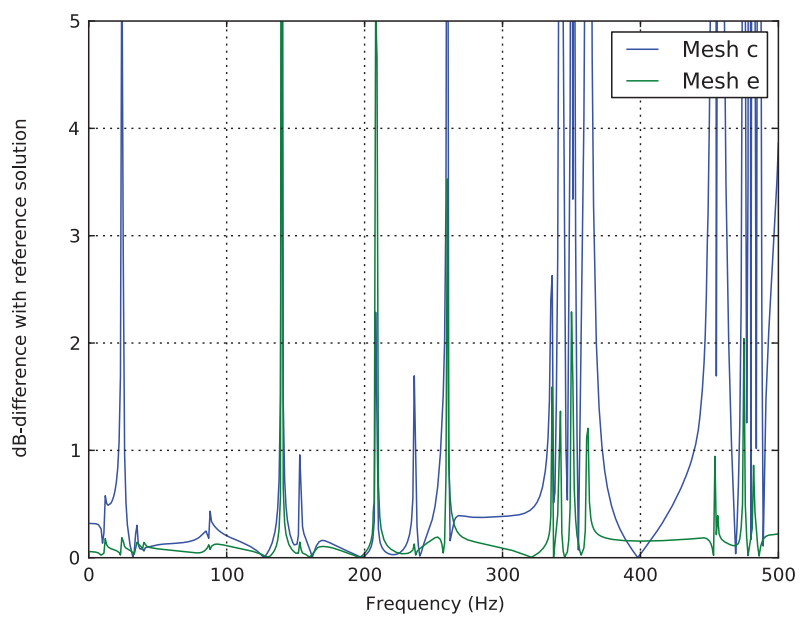

(c) $\mathrm{dB}$ difference with the reference solution

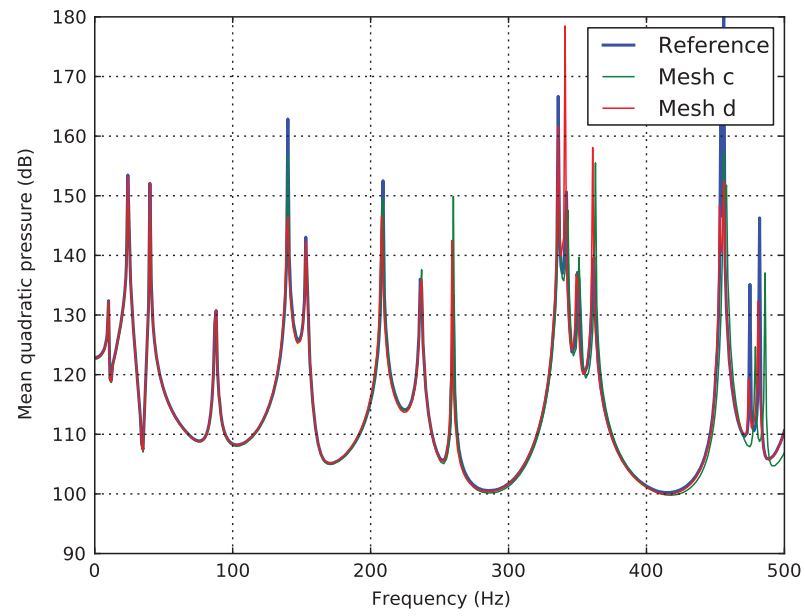

(b) Frequency response function, mesh $c$ and $d$

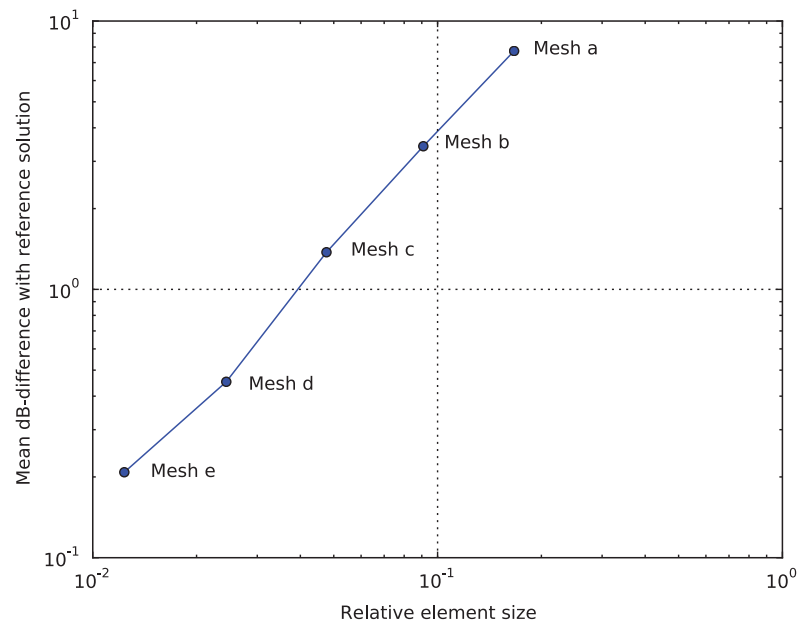

(d) Mean dB difference

Figure 17. Results of the immersed circular ring. (a) Frequency response function, meshes a and b; (b) frequency response function, meshes c and d; (c) dB difference with the reference solution; (d) mean $\mathrm{dB}$ difference.

it is arbitrarily cut up to a value of $5 \mathrm{~dB}$. To quantify the influence of the mesh size, a mean $\mathrm{dB}$ difference (denoted by $\zeta$ ) is computed for each mesh using the following simple formulae;

$$
\zeta=\frac{\sum \mathrm{dB} \text { difference }}{\text { Number of frequency steps }} .
$$

This mean $\mathrm{dB}$ difference (Table VII) is compared with the relative characteristic fluid mesh size to the radius of the circular structure in Figure 17(d). This last plot is more qualitative than quantitative; however, it gives a trend on how the fluid mesh size has to be compared with the curvature to give enough accurate results:

- The fluid element length has to be less than $1 / 20$ of the radius to have less than an average of $2 \mathrm{~dB}$ difference ( $c, d$, and $e$ meshes).

- A fluid element length larger than around 1/5 gives inaccurate results ( $a$ mesh).

The fluid mesh has thus to be prepared before the parametric study such that it is fine enough in the area where large structure curvatures are supposed to be.

\subsection{Application to an industrial-like problem: an aircraft cabin}

A cavity containing one or two separate flexible structures is considered. The geometry and the dimensions of the cavity and the structures are described in Figure 18 and in Table VIII. This case 


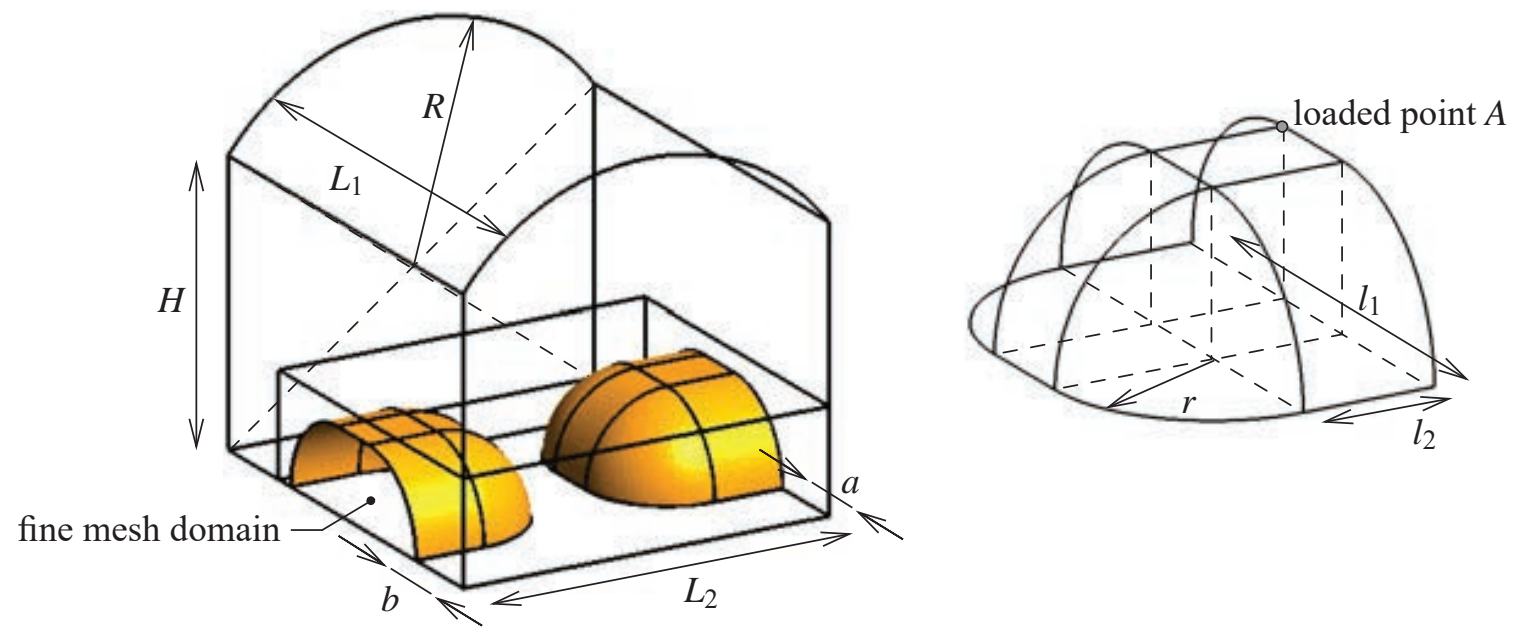

Figure 18. Aircraft cabin geometry.

Table VIII. Data of the aircraft cabin.

\begin{tabular}{lccc}
\hline & Fluid & \multicolumn{2}{c}{ Structure } \\
\hline$L_{1}(\mathrm{~m})$ & 3 & $E(\mathrm{~Pa})$ & $70000 \times 10^{6}$ \\
$L_{2}(\mathrm{~m})$ & 2.7 & $v$ & 0.27 \\
$H(\mathrm{~m})$ & 2 & $\rho_{S}\left(\mathrm{~kg} \cdot \mathrm{m}^{-3}\right)$ & 2700 \\
$R$ & $1 / 2 \sqrt{H^{2}+L_{2}^{2}}$ & $e$, thickness $(\mathrm{m})$ & $6 \times 10^{-3}$ \\
$c_{0}\left(\mathrm{~m} \cdot \mathrm{s}^{-1}\right)$ & 340 & $r(\mathrm{~m})$ & 0.6 \\
$\rho_{F}\left(\mathrm{~kg} \cdot \mathrm{m}^{-3}\right)$ & 1.2 & $l_{1}(\mathrm{~m})$ & 1.6 \\
& & $l_{2}(\mathrm{~m})$ & 0.5 \\
\hline
\end{tabular}

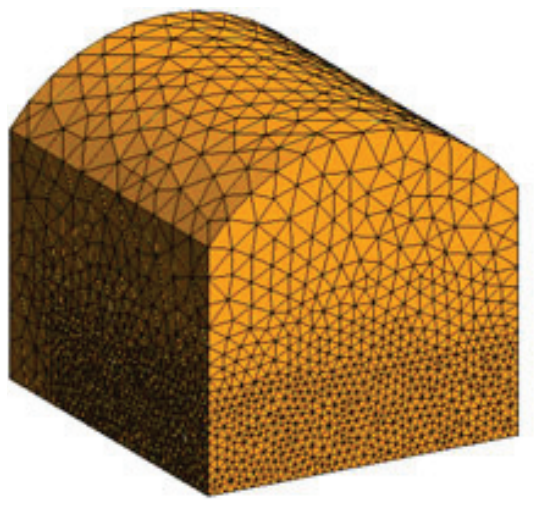

(a) Fluid mesh, 121789 tetrahedra and 20832 nodes

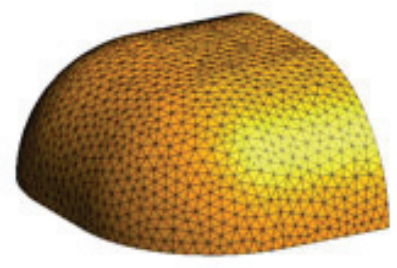

(b) Structure mesh, 2228 triangles and 1168 nodes

Figure 19. Fluid and structure meshes of the aircraft cabin. (a) Fluid mesh, 121,789 tetrahedra and 20,832 nodes; (b) structure mesh, 2228 triangles and 1168 nodes.

aims at being representative of an aircraft cabin with seats. The structures are simply supported (three fixed translations) on their bottom edges. One structure is loaded by an harmonic force of amplitude 1 in the three directions (total norm is $\sqrt{3}$ ) at point $A$. The frequency range is 30 to $100 \mathrm{~Hz}$. Three configurations are studied:

- Configuration $a$ : one structure, $a=0.6 \mathrm{~m}$.

- Configuration $b$ : one structure, $a=0.2 \mathrm{~m}$.

- Configuration $c$ : two opposite symmetric structures, $a=0.6 \mathrm{~m}$ and $b=0.6 \mathrm{~m}$.

The used meshes for the fluid and the structures are shown in Figure 19. For each configuration, the enriched elements are plotted in Figure 20, which shows that the restriction on the fluid element length around the structure (see Section 5.2) is fulfilled. 


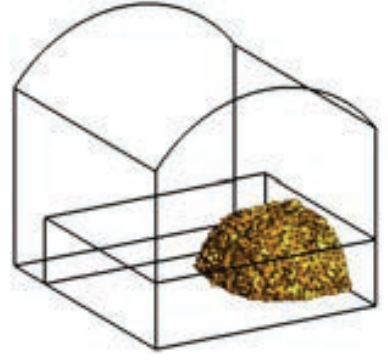

(a) Configuration $a$

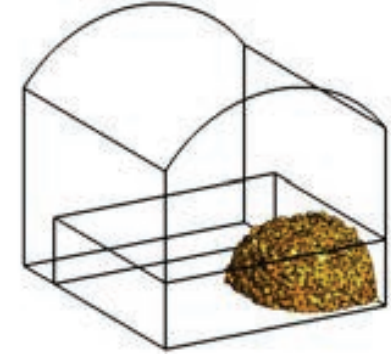

(b) Configuration $b$

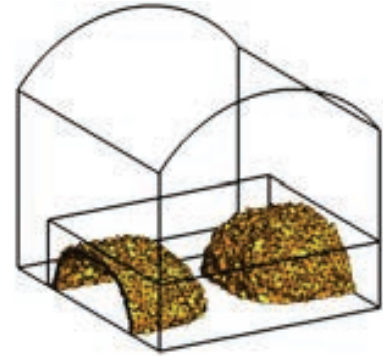

(c) Configuration $c$

Figure 20. Enriched elements for the three configurations of the aircraft cabin. (a) Configuration a; (b) configuration b; (c) configuration $\mathrm{c}$.

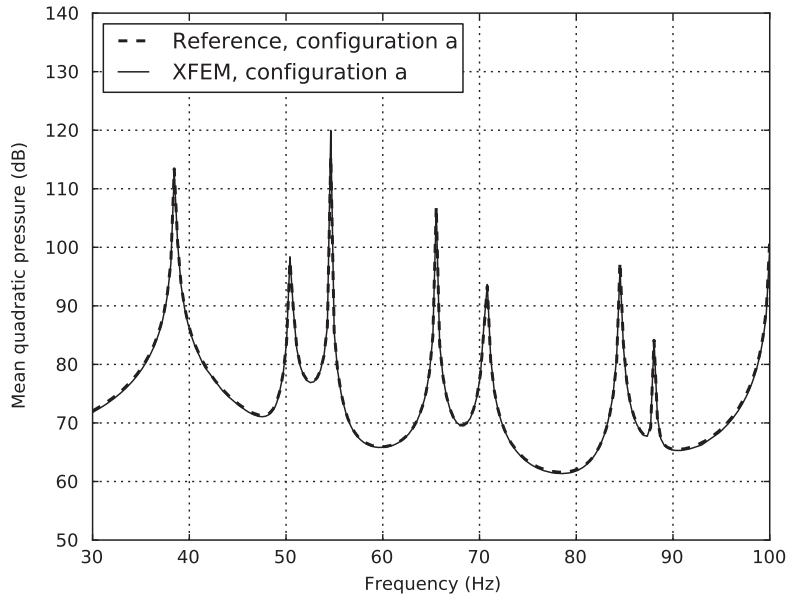

(a) FRF, configuration $a$

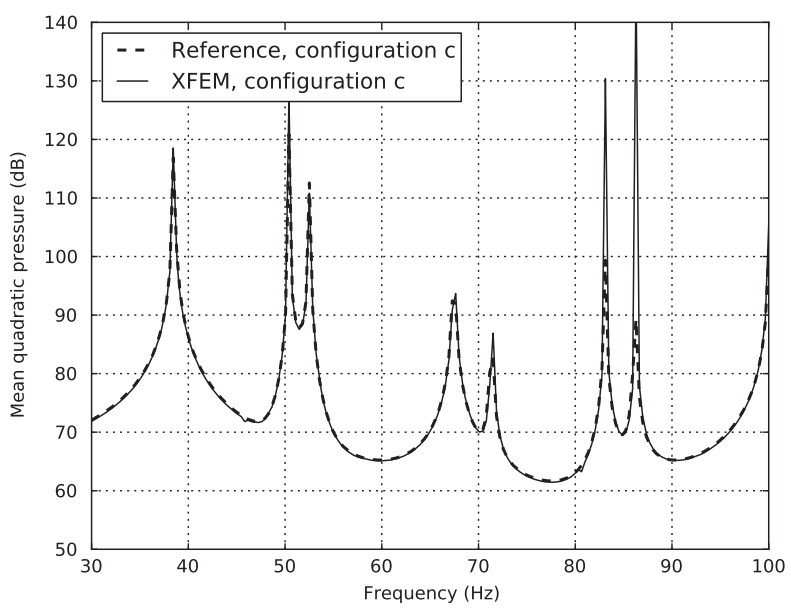

(c) FRF, configuration $c$

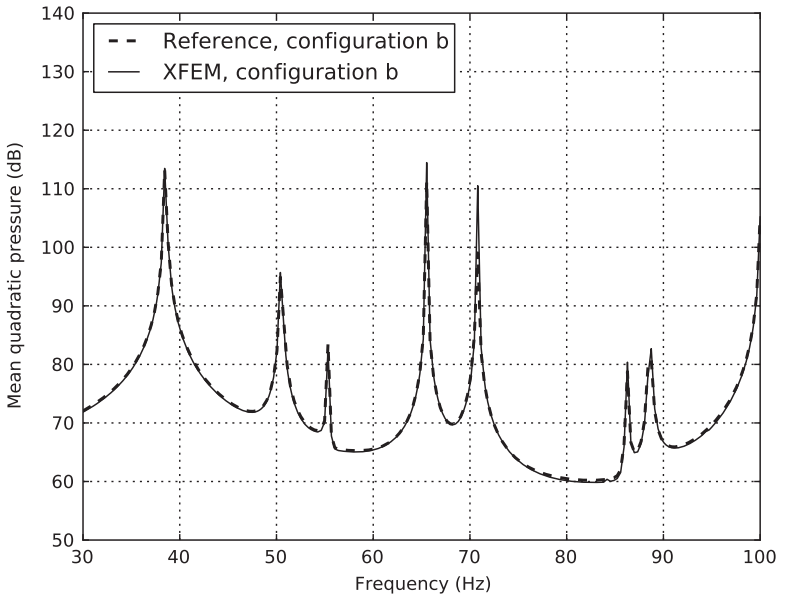

(b) FRF, configuration $b$

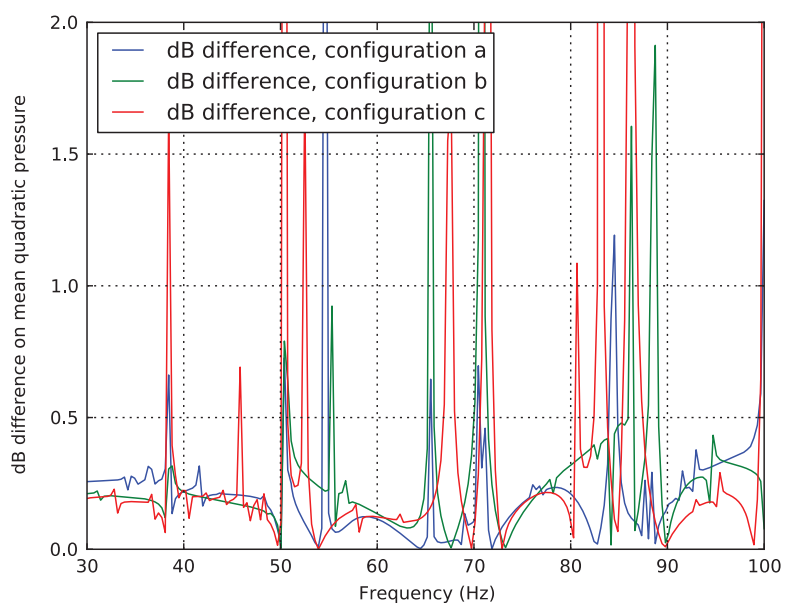

(d) dB difference

Figure 21. Frequency response function of the aircraft cabin. (a) FRF, configuration a; (b) FRF, configuration b; (c) FRF, configuration c; (d) dB difference.

The frequency response for each configuration as well as the $\mathrm{dB}$ difference with a reference solution obtained with a compatible mesh are plotted in Figure 21(a), 21(b), and 21(c) for configurations $a, b$, and $c$, respectively. The $\mathrm{dB}$ difference is less than $0.5 \mathrm{~dB}$ in this frequency range for the three configurations apart around the resonances.

For each configuration, a representation of the pressure field and the structural displacement is plotted in Figure 22 for frequencies close to resonances. For configuration $c$ at the $86.3 \mathrm{~Hz}$ resonance (Figure 22(f)), whereas only one structure is loaded by an external force, the second structure vibrates at the same displacement level as the first one: the coupling between the two structures is realized by the acoustic fluid. 


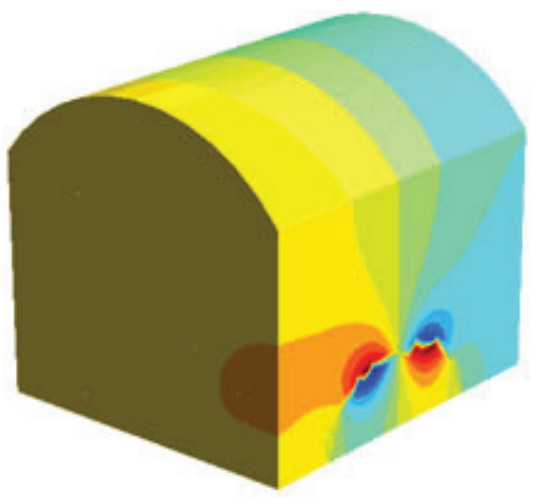

(a) Configuration $a$, fluid pressure, $50.4 \mathrm{~Hz}$

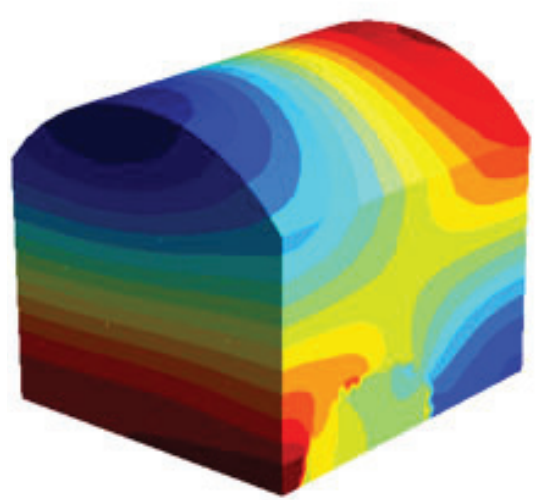

(c) Configuration $b$, fluid pressure, $88.7 \mathrm{~Hz}$

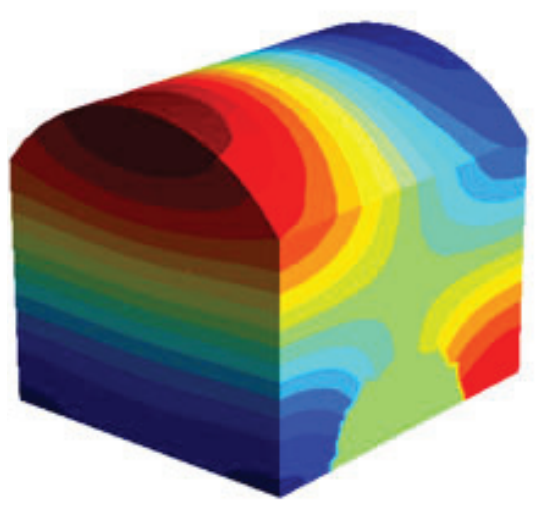

(e) Configuration $c$, fluid pressure, $86.3 \mathrm{~Hz}$

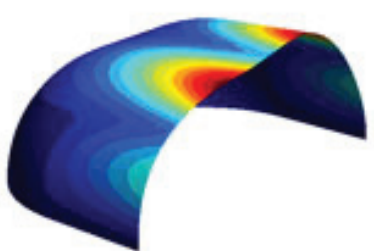

(b) Configuration $a$, structure displacement, $50.4 \mathrm{~Hz}$

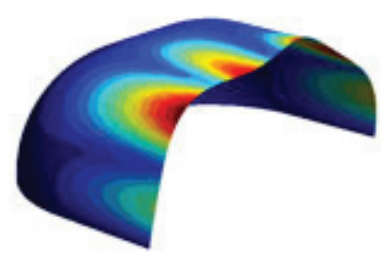

(d) Configuration $b$, structure displacement, $88.7 \mathrm{~Hz}$

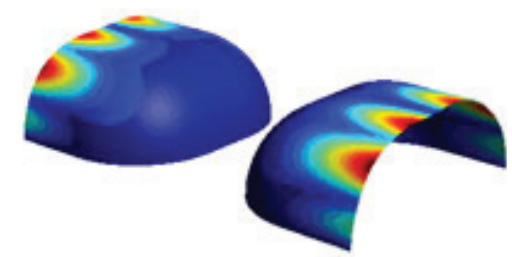

(f) Configuration $c$, structure displacement, $86.3 \mathrm{~Hz}$

Figure 22. Pressure field and structure displacement for the three configurations close to a chosen resonance. (a) Configuration a, fluid pressure, $50.4 \mathrm{~Hz}$; (b) configuration a, structure displacement, $50.4 \mathrm{~Hz}$; (c) configuration b, fluid pressure, $88.7 \mathrm{~Hz}$; (d) configuration b, structure displacement, $88.7 \mathrm{~Hz}$;

(e) configuration c, fluid pressure, $86.3 \mathrm{~Hz}$; (f) configuration c, structure displacement, $86.3 \mathrm{~Hz}$.

This last case demonstrates the ability and the flexibility of the method to deal with more complex problems where one or several structures are placed arbitrarily in an acoustic cavity.

\section{CONCLUSIONS}

The proposed method aims at avoiding remeshing process when performing a parametric study of different structure positions in an acoustic cavity. The structures are considered with no thickness in the fluid domain, and the fluid is supposed to be present on both sides of the structures. Therefore, the structures introduce pressure discontinuities through the fluid-structure interfaces. The XFEM 
is used because it is well suited to model such discontinuities inside elements, allowing to keep the same fluid mesh for all the structure positions in the cavity. The pressure enrichment is chosen as the Heaviside function on the basis of the signed distance to the structures.

The implementation is carried out in three dimension using four-node tetrahedral elements for the fluid and three-node discrete Kirchhoff triangle shell elements for the thin structures. A first application involving a plane structure in a parallelipipedic cavity shows the good ability of the method to deal with a plane surface of discontinuity. Moreover, several positions of the structures are computed, keeping the same fluid mesh for the whole parametric study.

A second case, made of a circular structure immersed in a thin squared fluid domain, enables studying the convergence of the method in terms of number of elements needed to represent correctly the fluid-structure interface. It turns out that the length of a fluid element has to be smaller than $1 / 10$ of the curvature radius of the structure around the interface. The fluid mesh has then to be built a priori with this condition according to the possible positions of the structures in the parametric study. A classical compatible mesh method would have the same restriction, but a mesh would have to be made for each configuration. This restriction could be less severe if the used fluid element approximation was be quadratic (10-node tetrahedral elements for instance), but for simplicity reasons, it has not been tested in this first work.

Finally, an industrial-like problem is studied, taking care of the previous recommendations for the fluid mesh. Three structure configurations are computed involving one or two structures. Results are in good agreement with reference solutions, showing that the developed approach is well suited for complex problems close to industrial applications.

\section{REFERENCES}

1. Cherng JG, Gang Y, Bonhard RB, French M. Characterization and validation of acoustic cavities of automotive vehicles. IMAC-XX, Conference on Structural Dynamics, Los Angeles CA, 2002.

2. Melenk J, Babuška JI. The partition of unity finite element method: basic theory and applications. Computer Methods in Applied Mechanics and Engineering 1996; 139(1-4):289-314.

3. Moës N, Dolbow J, Belytschko T. A finite element method for crack growth without remeshing. International Journal for Numerical Methods in Engineering 1999; 46(1):131-150.

4. Sukumar N, Chopp DL, Mos N, Belytschko T. Modeling holes and inclusions by level sets in the extended finite-element method. Computer Methods in Applied Mechanics and Engineering 2001; 190(46-47):6183-6200.

5. Legay A, Wang H, Belytschko T. Strong and weak arbitrary discontinuities in spectral finite elements. International Journal for Numerical Methods in Engineering 2005; 64(8):991-1008.

6. Duddu R, Bordas S, Chopp D, Moran B. A combined extended finite element and level set method for biofilm growth. International Journal for Numerical Methods in Engineering 2008; 74(5):848-870.

7. Legay A, Chessa J, Belytschko T. An Eulerian-Lagrangian method for fluid-structure interaction based on level sets. Computer Methods in Applied Mechanics and Engineering 2006; 195(17-18):2070-2087.

8. Zilian A, Legay A. The enriched space-time finite element method (EST) for simultaneous solution of fluid-structure interaction. International Journal for Numerical Methods in Engineering 2008; 75(3):305-334.

9. Gerstenberger A, Wall WA. An extended finite element method/Lagrange multiplier based approach for fluid-structure interaction. Computer Methods in Applied Mechanics and Engineering 2008; 197(19-20):1699-1714.

10. Wang H, Chessa J, Liu WK, Belytschko T. The immersed/fictitious element method for fluid-structure interaction: volumetric consistency, compressibility and thin members. International Journal for Numerical Methods in Engineering 2008; 74(1):32-55.

11. Wang X, Liu WK. Extended immersed boundary method using FEM and RKPM. Computer Methods in Applied Mechanics and Engineering 2003; 193(12-14):1305-1321.

12. Zhang L, Gerstenberger A, Wang X, Liu WK. Immersed finite element method. Computer Methods in Applied Mechanics and Engineering 2004; 193(21-22):2051-2067.

13. Laure P, Megally A, Coupez T. Collision strategy for the direct simulation of moving fibers in viscous fluid. In International Conference on Computational Methods for Coupled, Papadrakakis M, On̆ate E, Schrefler B (eds), Problems in Science and Engineering, 2005.

14. Allard J-F. Propagation of Sound in Porous Media: Modelling Sound Absorbing Materials. Elsevier Applied Science: London, New York, 1993.

15. Rumpler R, Legay A, Deü J-F. Performance of a restrained-interface substructuring FE model for reduction of structural-acoustic problems with poroelastic damping. Computers and Structures 2011; 89(23-24):2233-2248.

16. Davidsson P, Sandberg G. A reduction method for Structure-acoustic and poroelastic-acoustic problems using interface-dependent Lanczos vectors. Computer Methods in Applied Mechanics and Engineering 2006; 195(17-18):1933-1945. 
17. Deü J-F, Larbi W, Ohayon R. Vibration and transient response of structural acoustic interior coupled systems with dissipative interface. Computer Methods in Applied Mechanics and Engineering 2008; 197(51-52):4894-4905.

18. Bermúdez A, Rodríguez R. Modelling and numerical solution of elastoacoustic vibrations with interface damping. International Journal for Numerical Methods in Engineering 1999; 46(10):1763-1779.

19. Legay A, Zilian A, Janssen C. A rheological interface model and its space-time finite element formulation for fluid-structure interaction. International Journal for Numerical Methods in Engineering 2011; 86(6):667-687.

20. Rao MD. Recent applications of viscoelastic damping for noise control in automobiles and commercial airplanes. Journal of Sound and Vibration 2003; 262(3):457-474.

21. Bobillot A, Balmes E. Iterative techniques for eigenvalue solutions of damped structures coupled with Fluids. AIAA-2002-1391. 43rd AIAA/ASME/ASCE/AHS/ASC Structures, Structural Dynamics, and Materials Conference, Denver, Colorado, April 22-25, 2002.

22. Rouleau L, De J-F, Legay A, Sigrist J-F. Vibro-acoustic study of a viscoelastic sandwich ring immersed in water. Journal of Sound and Vibration 2012; 331(3):522-539.

23. Morand HJ-P, Ohayon R. Fluid Structure Interaction. John Wiley \& Sons: Chichester, 1995. ISBN: 978-0-47194459-1.

24. Stricklin JA, Haisler W, Tisdale P, Gunderson R. A rapidly converging triangular plate element. AIAA Journal 1969; 7(1):180-181.

25. Dhatt G. An efficient triangular shell element. AIAA Journal 1970; 8(11):2100-2102.

26. Batoz J-L, Bathe K-J, Ho L-W. A study of three-node triangular plate bending elements. International Journal for Numerical Methods in Engineering 1980; 15(12):1771-1812.

27. Batoz JL, Dhatt G. Modélisation des structures par éléments finis, HERMES; Vol. 3, Coques, 1992.

28. Ventura G. On the elimination of quadrature subcells for discontinuous functions in the extended finite-element method. International Journal for Numerical Methods in Engineering 2006; 66(5):761-795.

29. Geuzaine C, Remacle J-F. Gmsh: A 3-D finite element mesh generator with built-in pre- and post-processing facilities. International Journal for Numerical Methods in Engineering 2009; 79(11):1309-1331. 